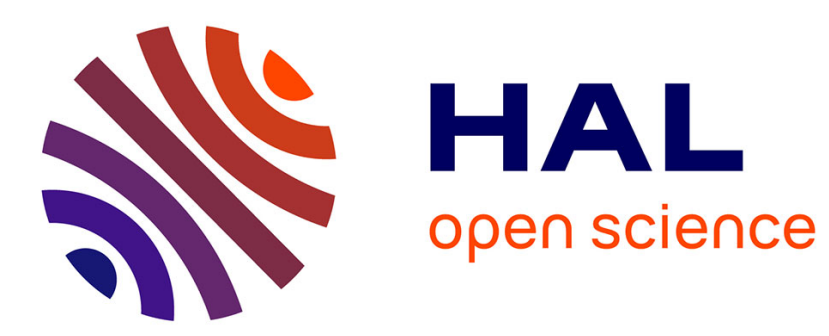

\title{
Motion from point matches: multiplicity of solutions
}

Olivier Faugeras, Steve Maybank

\section{To cite this version:}

Olivier Faugeras, Steve Maybank. Motion from point matches: multiplicity of solutions. [Research Report] RR-1157, INRIA. 1990. inria-00075401

\section{HAL Id: inria-00075401 https://hal.inria.fr/inria-00075401}

Submitted on 24 May 2006

HAL is a multi-disciplinary open access archive for the deposit and dissemination of scientific research documents, whether they are published or not. The documents may come from teaching and research institutions in France or abroad, or from public or private research centers.
L'archive ouverte pluridisciplinaire HAL, est destinée au dépôt et à la diffusion de documents scientifiques de niveau recherche, publiés ou non, émanant des établissements d'enseignement et de recherche français ou étrangers, des laboratoires publics ou privés. 


\section{Rapports de Recherche}

\section{$N^{\circ} 1157$}

IVIVASOPULA ANTIPOLSS

\section{MOTION FROM POINT MATCHES : MULTIPLICITY OF SOLUTIONS}

insturtu $\mathbb{N a t i o n a l ~}$ de Recherene

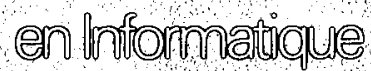

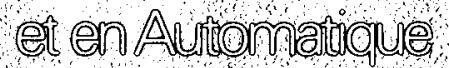

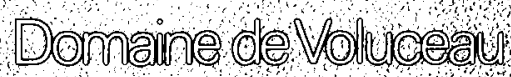
Rocouencoul [D:D: (0)

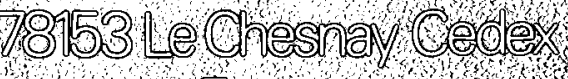

\section{Olivier D. FAUGERAS} Steve MAYBANK 


\title{
Motion from point matches: multiplicity of solutions*
}

\author{
Olivier D. Faugeras, \\ INRIA \\ BP 109 - 06561 Valbonne Cedex - FRANCE
}

\author{
Steve Maybank \\ Hirst Research Center, East Lane \\ Wembley, Middlesex, HA9 7PP - ENGLAND
}

\begin{abstract}
In this paper, we study the multiplicity of solutions of the motion problem. Given $n$ point matches between two frames, how many solutions are there to the motion problem ? We show that the maximum number of solutions is 10 when 5 point matches are available. This settles a question which has been around in the Computer Vision community for a while. We follow two tracks.

The first one attempts to recover the motion parameters by studying the essential matrix and has been followed by a number of researchers in the field. A natural extension of this is to use algebraic geometry to characterize the set of possible essential matrices. We present some new results based on this approach. The second one, based on projective geometry, dates from the previous century:

We show that the two approaches are compatible and yield the same result.

We then describe a computer implementation of the second approach that uses MAPLE, a language for symbolic computation. The program allows us to compute exactly the solutions for any configuration of 5 points. Some experiments are described.
\end{abstract}

\section{Mouvement à partir de points: nombre de solutions}

\begin{abstract}
Nous étudions dans cet article le nombre de solutions au problème du calcul du mouvement d'une caméra à partir de deux vues. Etant donnés n points appariés entre les deux images, combien de solution $\mathrm{y}$-a-t-il au problème du mouvement? nous montrons que le nombre maximum de solutions est 10 quand 5 appariements sont donnés. Ceci résotid une question qui agite la communauté de la Vision par Ordinateur depuis un certain temps. Dans notre travail, nous avons suivi deux approches:

Avec la première, nous calculons les paramètres du mouvement en étudiant la matrice essentielle. Cette approche a été suivie par un certain nombre de cluercheurs de notre discipline. Une généralisation naturelle de celle-ci est d'utiliser des méthodes de géométrie algébrique afin de caractériser l'ensemble des matrices essentielles possibles. Nous présentons ici quel ques résultats nouveaux obtenus grâce à cet.te approche. La seconde approche utilise la géométrije projective.

Nous montrons que les deux approches sont compatibles et fournissent des résultats identiques.

Nous décrivons une implantation de la seconde en MAPLE, un langage de calcul symbolique. Le programme nous permet de calculer exactement les solutions pour n'importe quelles configurations de 5 points. Nous décrivons quelques expériences réalisées avec ce système.
\end{abstract}

"This work was partially supported mider ESPRI'T contract P940 


\section{Introduction}

Given two images taken at different times by a camera moving in a static environment, we want to estimate the position and orientation of the camera with respect to its first position. Many approaches are possible and we consider in this paper only the approach consisting of identifying within the two images corresponding points, i.e. pairs of points which are images of the same physical point viewed from the two different positions.

Given a number of such pairs, the following questions may be asked:

1. can the position and orientation of the camera be recovered?

2. if yes, what is the minimal number of pairs of points necessary?

3. is there a unique solution to the motion?

Partial answers to these questions have been known for a while in the Computer Vision community. For example, it is known that given a sufficient number of pairs (greater than or equal to 5 ), the position and orientation of the camera can be recovered. If 8 pairs or more are given it can even be recovered by linear techniques [Lon81] unless the 3D points and the optical centers of the cameras fall on some special second degree surfaces. When they do fall on such surfaces, it is known [Lon87,May85,Hor87] that the solution is not unique, and that there may not be more than 3 different solutions to the motion problem.

Not much is known so far on what happens when the number of pairs is between 5 and 7 . We shed some light on these problems, and show in particular that when 5 pairs are given in general position, then the maximum number of solutions is 10 . "In general position" means that although some sets of 5 pairs are not compatible with exactly 10 camera motions, such sets form a negligibly small part of the space of all sets of 5 pairs of image correspondences.

It might be thought that studying these problems is a bit remote from the harsh reality of applied Computer Vision, but we believe that their solution is essential to understand the behaviour (stability or instability) of algorithms for recovering motion from point matches. It is the mathematical structure of the set of solutions that governs this behaviour, and only if we understand it thoroughly will we be able to design reliable systems for motion analysis.

In our investigation of the multiplicity of solutions to the motion problem we use two very different approaches. The first approach begins with a $3 \times 3$ matrix known as the essential matrix, which contains the information about camera motion recoverable from corresponding points in the two retinal planes. The set of essential matrices can be described by polynomial equations, thus the problem of recovering camera motion can be investigated using algebraic geometry.

The second approach is an older one based on projective geometry. We begin with the epipoles, which are the points of intersection of a line joining the two optical centres with the retinal planes. Lines in the retinal planes containing an epipole are called epipolar lines. Corresponding points in the two retinal planes define a homography or projective correspondence between the two sets of epipolar lines, which forms the basis of a method for recovering camera motion. It is at first sight difficult to include the rigidity constraint in a natural way. It turns out that this can be done using the device of the absolute conic.

The projective geometric approach is based on jdeas developed by Chasles [Cha55] and Joseph Kruppa. [Kru13]. Kruppa was an Austrian mathematician, who published his result in 1913; unfortunately, he found 11 solutions, rather than 10; a computer implemention of the projective geometry based approach allowed us to correct the error, and then to give a formal proof.

The second approach has two advantages over the first: the mathematics is more elementary, and it leads to more tractable polynomial constraints on the camera motion when only 5 image correspondences are given. However, these are early days, and it is not clear which approach will give the clearest insight in to the motion problem. 


\subsection{Organisation of the paper}

In section 2 we describe the framework for the motion problem and give some more details about our two approaches. In section 3 we derive some new properties of the essential matrix, and show that the essential matrices have to lie on a specific (real) algebraic manifold $\mathcal{M}$ defined by two equations of degrees 3 and 4 respectively. It turns out that in order to understand the problem, we have to abandon for a. while the set of real numbers and deal with complex numbers. In section 4 we present some results recently obtained by Demazure [Dem88] which completely characterize the structure of $\mathcal{M}$ over the complex numbers; $\mathcal{M}$ is defined by 9 equations of degree three; we show how to solve the apparent contradiction in the number of equations and cite the main result which is that the degree of $\mathcal{M}$ is 10 ; it follows that there are, in general, exactly 10 camera motions compatible with 5 pairs of corresponding points. We then show how the degree of the equations defining $\mathcal{M}$ can be used to derive very simply some results previously obtained by Longuet-Higgins ([Lon87,Lon88]), Maybank ([May85, May87]), and Horn ([Hor87]) on 3 being the maximum possible number of solutions when an infinite number of corresponding points are available.

In section 5 we develop the approach based on projective geometry which allows us to compute explicitly the 10 solutions from the 36 intersection of two sextic plane curves. The remaining 26 intersections do not yield solutions to the motion problem. In section 6 we describe a computer implementation which malies henvy use of symbolic computation.

Some concluding remarks are made in section 7 . We have two appendices: appendix A summarises sone properties of polynomials; and appendix B summarises the projective geometry necessary for Kruppa's method.

\subsection{Notation}

We use $\mathbf{a}, \mathbf{b}, \mathbf{c}, \ldots$ both for column vectors and for points in projective space $\mathcal{F}^{n}, n=1$, and we use $\mathbf{A}$, $\mathbf{B}, \mathbf{C}, \ldots$ for matrices or for points in $\mathcal{P}^{3}$ when no ambiguity is possible. The row vector corresponding to $\mathbf{a}$ is $\mathbf{a}^{\top}$, and the transpose of $\mathbf{A}$ is $\mathbf{A}^{\top}$. The trace of $\mathbf{A}$ is $\operatorname{Tr}(\mathbf{A})$ and, the determinant of $\mathbf{A}$ is $\operatorname{det}(\mathbf{A})$.

The coordinates of a vector $\mathrm{a}$ in $3 \mathrm{D}$ space are $\left(a_{1}, a_{2}, a_{3}\right)^{\top}$; if $\mathrm{a}$ is regarded as a point in $\mathcal{P}^{3}$ then the coordinates of a are $\left(a_{1}, a_{2}, a_{3}, a_{4}\right)^{\top}$. The line joining two points $\mathrm{a}, \mathrm{b}$ is denoted $\mathrm{by}\langle\mathrm{a}, \mathrm{b}\rangle$. The vector product of two vectors $\mathbf{a}, \mathbf{b}$ is $\mathbf{a} \wedge \mathbf{b}$, and the scalar or rot product of $\mathbf{a}, \mathbf{b}$ is $\mathbf{a}^{\top} \mathbf{b}$. We write $\mathbf{a}^{\top} \mathbf{a}=\mathbf{a}^{2}=\|\mathbf{a}\|^{2}$. The tensor product of $\mathbf{a}, \mathbf{b}$ is $\mathbf{a} \mathbf{b}^{\top}$. By definition, $\mathbf{a} \mathbf{b}^{\top}$ is the $3 \times 3$ matrix with $i, j$ th entry equal to $a_{i} b_{j}$.

We use $\{a, b ; c, d\}$ for the cross ratio of four points in $\mathcal{P}^{\mathrm{l}}$. A homography between points $\mathrm{a}, \mathrm{b}$ of $\mathcal{P}^{1}$ is denoted by a $\bar{\wedge}$ b. We use $\Omega$ for the absolute conic, and $\omega, \omega^{\prime}$ for the images of $\Omega$ in the two retinal planes (sec Appendix B). We use $\Phi, \Psi$ for quadratic transformations.

She symbol $\square$ marks the end of a proof.

\section{Statement of the problem}

We model our camera as a pinhole, as shown in Figure 1. Each point $\mathrm{M}$ in space gives rise to corresponding points $\mathbf{m}, \mathbf{m}^{\prime}$ in the first and sccond retinal, or image planes, where we assume that the correspondence $\mathbf{m}-\mathbf{m}^{\prime}$ is known. Each camera has an associated coordinate frame, $y_{1}, y_{2}, y_{3}$, in which the positions of the image points are measured. The $y_{3}$ axis is the optical axis and the retinal plane is parallel to the $y_{1}, y_{2}$ plane. We assume that the focal length $\|\mathrm{Cc}\|$ is equal to 1 .

In practice the camera projection is more complicated than the projection obtained from an ideal pinhole camera, however it is usually possible to calibrate the camera and thus find the image that would have been obtained from a pinhole camera. Thanks to this, the coordinates $y_{1}, y_{2}, y_{3}$ of a point $M$ in $3 \mathrm{D}$ space are the projective coordinates of its image $\mathrm{m}$. Points at infinity in the image plane are defined by $y_{3}=0$ (sée 


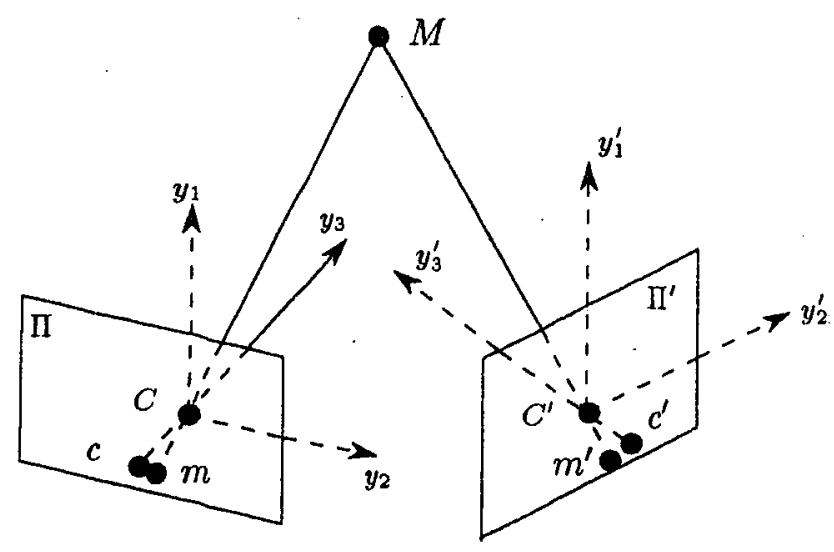

Figure 1: Geometry of the motion problem

appendix $\mathrm{B}$ ). Indeed, when $\mathbf{M}$ is in the $y_{1} y_{2}$ plane (called the focal plane), $<\mathbf{C M}>$ is parallel to the image plane.

The problem is now to obtain the camera motion from the image correspondences. Probably the oldest example of this problem is the version given by the French mathematician Chasles [Cha5.5]. We found a statement of the problem in a paper by Hesse [Hes63] who also produced an analytical solution:

"On donne dans le même plan deux systèmes de sept points chacun et qui se correspondent. Faire passer par chacun de ces systèmes un faiscean de sept rayons, de telle sorte que les deux faisceaux soient homographiques."1

A detailed analysis and solution of Chasles' problem can be found in [Stu69]. The final word on this is that there exist three solutions in general, real or complex, which can be found as a subset of the intersection of two planar cubics. Unfortunately this does not solve our particular problem since there is no guarantee that the corresponding transformation from the first camera to the second is a rigid displacement. In the following sections we describe two approaches to solving Chasles' problem which incorporate the rigidity constraint.

\subsection{Approach based on essential matrices}

Let the rotation from the first camera position to the second be $\mathbf{R}$, and let the translation be a vector $\mathbf{t}$ in the direction $\mathbf{C}$ to $\mathbf{C}^{\prime}$, where $\mathbf{C}, \mathbf{C}^{\prime}$ are the optical centers of the two cameras. The vector $\mathbf{t}$ is assumed to be non-zero. To fix ideas, we think of the camera as first unclergoing a rotation $\mathbf{R}$ and then undergoing a translation $\mathbf{t}$.

A point $M$ in $3 D$ space gives rise to image points $\mathbf{m}$ and $\mathbf{m}^{\prime}$ in the first and second retinal planes respectively. The problem is: given a number of corresponding pairs $\mathbf{m} \leftrightarrow \mathbf{m}^{\prime}$, to recover $\mathbf{R}$ and the direction of $t$.

Referring to Figure 1, it is clear that $\mathbf{m}$ and $\mathbf{m}^{\prime}$ are matches if and only if the three vectors $\mathbf{m}, \mathbf{m}^{\prime}$, and $\mathbf{t}$ are coplanar which we can write in the second coordinate system as:

$$
\mathbf{m}^{\top}\left(\mathbf{t} \wedge \mathbf{R} \mathbf{m}^{\prime}\right)=0
$$

\footnotetext{
1 "We are given, in a plane, two sets of seven points, in correspondence. For each set, find a point such that the two sets of seven lines they define are related by a collineation."
} 


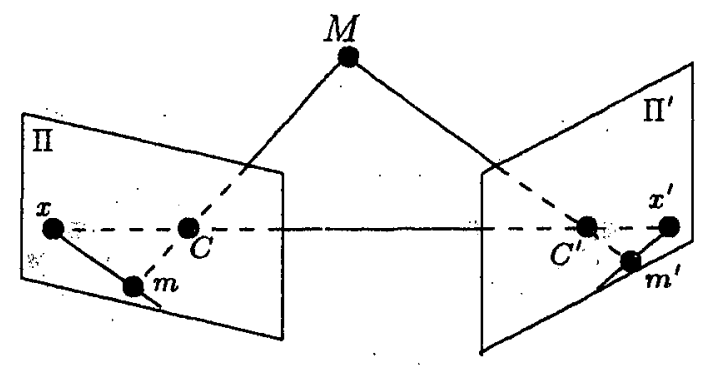

Figure 2: Epipolar geometry

We introduce the antisymmetric matrix $\mathrm{T}$ :

$$
\mathbf{T}=\left[\begin{array}{ccc}
0 & -t_{3} & t_{2} \\
t_{3} & 0 & -t_{1} \\
-t_{2} & t_{1} & 0
\end{array}\right]
$$

The matrix $\mathbf{T}$ is such that $\mathbf{T x}=\mathbf{t} \wedge \mathbf{x}$ for all vectors $\mathbf{x}$. Letting $\mathbf{E}=\mathbf{T R}$, equation (1) can be. rewritten as:

$$
\mathbf{m}^{T} \mathbf{E m}^{\prime}=0
$$

The matrix $\mathrm{E}$ is the essential matrix, first introduced by Longuet-Higgins in [Lon81].

There are in fact two rigid motions $t, \mathbf{R}$ and $\mathbf{t}^{\prime}, \mathbf{R}^{\prime}$ such that $\mathbf{E}=\mathbf{T} \mathbf{R}=\mathbf{T}^{\prime} \mathbf{R}^{\prime}$, where $\mathbf{t}$ and $\mathbf{t}^{\prime}$ are parallel and $\mathbf{R}, \mathbf{R}^{\prime}$ differ by a rotation of $180^{\circ}$ about $\mathbf{t}$. For the most part, we think of $\mathbf{t}, \mathbf{R}$ and $\mathbf{t}^{\prime}, \mathbf{R}^{\prime}$ as constituting a single solution to the motion problem, however it is necessary to consider both $t, R$ and $t^{\prime}$, $\mathbf{R}^{\prime}$ when checking the physical feasibility of solutions to the motion problem, as described in section 6 .

\subsection{Approach based on epipolar geometry}

When the problem of obtaining camera motion from image correspondences arose during the 19th century it was tackled using the projective geometry of the epipolar lines. We again refer to the camera geometry of Figure 1. By definition, the epipoles $\mathbf{x}$ and $\mathbf{x}^{\prime}$ are the intersections of $\left\langle\mathbf{C}, \mathbf{C}^{\prime}\right\rangle$ with the retinal planes; these points play an important role in Computer Vision since they form the basis of the so-called epipolar constraint, heavily used in stereo, for example. The epipolar constraint simply states that given a point $M$ in 3D space with an image $\mathbf{m}$ in the first retinal plane and $\mathbf{m}^{\prime}$ in the second retinal plane then the epipolar plajie $\left\langle\mathbf{C}, \mathbf{C}^{\prime}, \mathbf{M}\right\rangle$ intersects the retinal planes along the lines $\langle\mathbf{x}, \mathbf{m}\rangle$ and $\left\langle\mathbf{x}^{\prime}, \mathbf{m}^{\prime}\right\rangle$. Therefore, given a point $\mathbf{m}\left(\mathbf{m}^{\prime}\right)$. in the first (second) image, its image $\mathbf{m}^{\prime}(\mathbf{m})$ in the second: (first) image lies on the corresponding epipolar line. The geometry of epipolar lines is shown in figure 2.

The two sets of epipolar lines are pencils of lines around the epipoles $\mathbf{x}$ and $\mathbf{x}^{\prime}$ (see appendix B) which are related by a linear projective transformation, a collineation.

We can prove linearity directly since corresponding epipolar lines arise from the intersection of a single epipolar plane with the two image planes. Alternatively, we can construct a proof using the rigid motion $R, t$ of the previous section which induces the correspondence between epipolar lines. It follows from the definition of $R, t$ that $x=t$, and $x^{\prime}=-R^{\top} t$. On rotating the camera by $R$ the epipolar lines through $x$ are transformed linearly to epipolar lines through the point $\mathrm{x}^{\prime}$. On now translating the camera by $t$, the images of points move along lines passing through $t$, thus the translation acts as the identity on the lines through $t$. This is just the well known property of the focus of expansion. It follows that the correspondence between epipolar lines is induced entirely by $\mathbf{R}$, thus it is linear as claimed. 


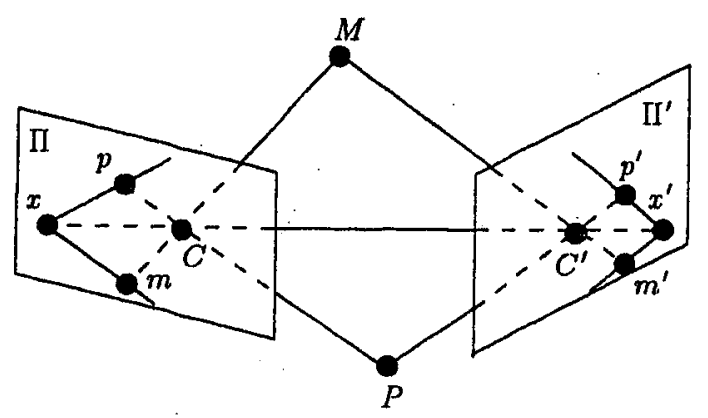

Figure 3: The angles $(x C m, x C p)$ and $\left(x^{\prime} C^{\prime} m^{\prime}, x^{\prime} C^{\prime} p^{\prime}\right)$ are equal

It follows from the linearity of the correspondence of epipolar lines that if we consider four epipolar planes $\Psi_{1}, \Psi_{2}, \Psi_{3}, \Psi_{4}$ and the corresponding epipolar lines $l_{1}, l_{1}^{\prime}, l_{2}, l_{2}^{\prime}, l_{3}, l_{3}^{\prime}$, and $l_{4}, l_{4}^{\prime}$, then the cross-ratios $\left\{l_{1}, l_{2} ; l_{3}, l_{4}\right\}$ and $\left\{l_{1}^{\prime}, l_{2}^{\prime} ; l_{3}^{\prime}, l_{4}^{\prime}\right\}$ are equal. For the definition of the cross-ratio of four lines see [SK52, chapter 4] and appendix $B$.

A correspondence between one dimensional projective spaces preserving cross ratio is known as a homography, and is denoted by $\bar{\lambda}$. If $\mathbf{m} \leftrightarrow \mathbf{m}^{\prime}$ are corresponding points then we write $\langle\mathbf{m}, \mathbf{x}\rangle \bar{\lambda}\left\langle\mathbf{m}^{\prime}, \mathbf{x}^{\prime}\right\rangle$.

In the original statement of Chasles' problem we are given 7 point correspondences in two planes from which we can deduce a number of pairs of epipoles. From each pair of epipoles we can infer a projective transformation of $3 D$ space that brings the pair $(\mathbf{C}, \Pi)$ onto $\left(\mathbf{C}^{\prime}, \Pi^{\prime}\right)$, where $\Pi$, $\Pi^{\prime}$ are the two retinal planes; however, there is no guarantee that this projective transformation is a rigid displacement. The introduction of the rigidity constraint enables us to solve the problem with 5 correspondences rather than 7 correspondences, but at the cost of obtaining 10 solutions rather than 3 solutions.

To enforce rigidity we can look at figure 3 , and notice that by construction, in the case of a rigid camera. motion, the plane angles $(x C m, x C p)$ and $\left(x^{\prime} C^{\prime} m^{\prime}, x^{\prime} C^{\prime} p^{\prime}\right)$ are equal. According to the projective definition of the angle between two planes given in Appendix B, we must introduce the absolute conic, $\Omega$ (see section B). Considering the images $\omega$ and $\omega^{\prime}$ of $\Omega$ in the two retinal planes, let $u, v$ be the two tangents from $\mathrm{x}$ to $\omega$ and let $u^{\prime}, v^{\prime}$ be the two tangents from $\mathbf{x}^{\prime}$ to $\omega^{\prime}$. If we want the equality of the two previous plane angles, a necessary condition is that the cross-ratios $\{\langle\mathbf{x}, \mathbf{m}\rangle,\langle\mathbf{x}, \mathbf{p}\rangle ; u, v\}$ and $\left\{\left\langle\mathbf{x}^{\prime}, \mathbf{m}^{\prime}\right\rangle,\left\langle\mathbf{x}^{\prime}, \mathbf{p}^{\prime}\right\rangle ; u^{\prime}, v^{\prime}\right\}$ are equal. This implies that these tangents correspond to each other in the previous collineation. This is shown in Figure 4.

It turns out that the precise correspondence of the tangents is immaterial, in that we could have either $u \pi u^{\prime}, v \pi v^{\prime}$ or $u \pi v^{\prime}, v \bar{\lambda} u u^{\prime}$. For this reason we will refer to corresponding pairs of tangents without making the correspondence explicit.

Define the lines $l_{a}, l_{a^{\prime}}, \ldots$ by $l_{a}=\langle\mathbf{x}, \mathbf{a}\rangle, l_{a^{\prime}}=\left\langle\mathbf{x}^{\prime}, \mathbf{a}^{\prime}\right\rangle, \ldots$ the problem is then as follows:

Given five point correspondences in $\Pi$ and $\Pi^{\prime}$ and the images $\omega$ and $\omega^{\prime}$ of the absolute conic $\Omega$, find two points $\mathbf{x}$ and $\mathbf{x}^{\prime}$ in $I I$ and $\Pi^{\prime}$ respectively such that if we denote by $w$ (resp. $w^{\prime}$ ) the pajrs of tangents from $\mathbf{x}\left(\right.$ resp. $\left.\mathbf{x}^{\prime}\right)$ to $\omega\left(\right.$ resp. $\left.\omega^{\prime}\right)$, the seven correspondences $l_{a} \leftrightarrow l_{a^{\prime}}, l_{b} \leftrightarrow l_{b^{\prime}}, l_{c} \leftrightarrow l_{c^{\prime}}, l_{d} \leftrightarrow l_{d^{\prime}}, l_{e} \leftrightarrow l_{e^{\prime}}, l_{w}-l_{w^{\prime}}$ are homographic $\left(l_{w} \hookleftarrow l_{w}\right.$, includes both tangents). We denote this correspondence by:

$$
\mathbf{x}(\mathbf{a}, \mathbf{b}, \mathbf{c}, \mathbf{d}, \mathbf{e}, \omega) \pi \mathbf{x}^{\prime}\left(\mathbf{a}^{\prime}, \mathbf{b}^{\prime}, \mathbf{c}^{\prime}, \mathbf{d}^{\prime}, \mathbf{e}^{\prime}, \omega^{\prime}\right)
$$

\section{Properties of the essential matrix}

We begin with the approach to Chasles' problem based on essential matrices. In this section we derive a number of results from which we obtain two polynomial constrajnts specifying exactly the set, of essential 


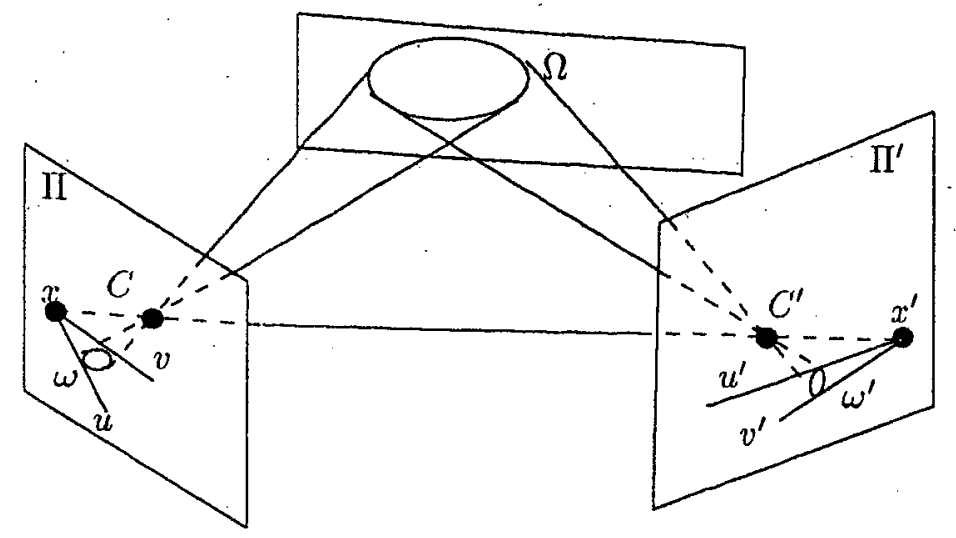

Figure 4: The images of the absolute conic $\Omega$

matrices with real entries.

Proposition 1 The essential matrix $\mathbf{E}=\mathbf{T R}$ satisfies $\mathbf{E}^{\top} \mathbf{t}=0$.

Proof: $E^{\top}=R^{\top} T^{\top}=-R^{\top} T$. Therefore $E^{\top} t=-R^{\top}(T t)=-R^{\top}(t \wedge t)=0$

One consequence of Proposition 1 is that the rank of the matrix $E$ is less than or equal to 2, and therefore, $\operatorname{det}(E)=0$.

Proposition 2 The essential matrix: $\mathrm{E}=\mathrm{TR}$ is such that $\mathrm{EE}^{\top}$ depends only on the translation vector $\mathrm{t}$.

Proof : Clearly, we have:

$$
\mathrm{EE}^{\top}=\operatorname{TRR}^{\top} \mathbf{T}^{\top}=-\mathbf{T}^{2}=\left[\begin{array}{ccc}
t_{3}^{2}+t_{2}^{2} & -t_{1} t_{2} & -t_{1} t_{3} \\
-t_{2} t_{1} & t_{1}^{2}+t_{3}^{2} & -t_{2} t_{3} \\
-t_{3} t_{1} & -t_{3} t_{2} & t_{2}^{2}+t_{1}^{2}
\end{array}\right]
$$

$\square$ We note that

$$
E E^{\top}=-T^{2}=\left(t^{\top} t\right) \mathbf{I}-t^{\top}
$$

An important consequence of Proposition 2 is that the essential matrix $\mathbf{E}$ has two equal singular values [GVL83]. In detail, let $U$ be a rotation matrix such that $U t=(1,0,0)^{\top}$ ( $t$ can be assumed of unit length since we know that it can be recovered only up to a scale factor). Then

$$
\mathbf{U T T}^{\top} \mathbf{U}^{\top}=\mathbf{I}-(\mathbf{U} \mathbf{t})(\mathbf{U} \mathbf{t})^{\top}=\left[\begin{array}{lll}
0 & 0 & 0 \\
0 & 1 & 0 \\
0 & 0 & 1
\end{array}\right] \equiv \mathbf{A}
$$

thus

$$
\mathrm{UEE}^{\top} \mathbf{U}^{\top}=\mathbf{U T R R} \mathbf{R}^{\top} \mathbf{T}^{\top} \mathbf{U}^{\top}=\mathbf{A}
$$

The matrices $\mathbf{E}$ and $\mathbf{A}$ thus have the same singular values. It is interesting to note that the reverse is true. More precisely we have the theorem ${ }^{2}$ :

\footnotetext{
${ }^{2}$ The proof of this theorem was found by Huang and Faugeras, and independently by Maybank. The molivalion for the theorem came from a conversation with Braccini (University of Genoa).
} 
Theorem 1 A real $3 \times 3$ matrix $\mathbf{E}$ can be decomposed as the product $\mathbf{T R}$ of an antisymmetric matrix $\mathrm{T}$ and a rotation matrix $\mathbf{R}$ if and only if it has one singular value equal to 0 and the other two singular values equal to each other.

Proof : We have just proved the only if part. Let us prove the if part. If $E$ has one singular value equal to zero and two singular values equal to $\sigma \neq 0$, then there exists a rotation matrix $U$ such that:

$$
\mathrm{EE}^{\top}=\mathrm{U}^{\top}\left[\begin{array}{ccc}
0 & 0 & 0 \\
0 & \sigma^{2} & 0 \\
0 & 0 & \sigma^{2}
\end{array}\right] \mathrm{U}
$$

Thus

$$
\left(\sigma^{-1} \mathrm{UE}\right)\left(\sigma^{-1} \mathrm{UE}\right)^{\top}=\mathbf{A}
$$

Let $\mathbf{F}=\sigma^{-1} \mathbf{U E}$, and let $\mathbf{f}_{1}^{\top}, \mathbf{f}_{2}^{\top}$, and $\mathbf{f}_{3}^{\top}$ be the row vectors of $\mathbf{F}$. From equation (3) we have:

$$
\begin{array}{lll}
\mathbf{f}_{1}^{\top} \mathbf{f}_{1}=0 & \text { and therefore } & \mathbf{f}_{1}=0 \\
\mathbf{f}_{2}^{\top} \mathbf{f}_{2}=\mathbf{f}_{3}^{\top} \mathbf{f}_{3}=1 \quad \text { and } & \mathbf{f}_{2}^{\top} \mathbf{f}_{3}=0
\end{array}
$$

Matrix $\mathbf{F}$ is therefore "almost" orthogonal. It is easy to construct an orthogonal matrix $\mathrm{F}^{\prime}$ such that $\mathrm{FF}^{\prime \top}=\mathrm{A}$. Indeed, we can choose the row vectors $\mathrm{f}_{1}^{\prime \top}, \mathrm{f}_{2}^{\prime \top}$, and $\mathrm{f}_{3}^{\prime \top}$ of $\mathrm{F}^{\prime}$ such that:

$$
\begin{aligned}
& \mathbf{f}_{1}^{\prime}=\mathbf{f}_{2} \wedge \mathbf{f}_{3} \\
& \mathbf{f}_{2}^{\prime}=\mathbf{f}_{2} \\
& \mathbf{f}_{3}^{\prime}=\mathbf{f}_{3}
\end{aligned}
$$

We can therefore write:

$$
\mathrm{F}=A F^{\prime}
$$

We notice that matrix $\mathbf{A}$ can be written:

$$
\mathbf{A}=\left[\begin{array}{ccc}
0 & 0 & 0 \\
0 & 0 & -1 \\
0 & 1 & 0
\end{array}\right]\left[\begin{array}{ccc}
1 & 0 & 0 \\
0 & 0 & 1 \\
0 & -1 & 0
\end{array}\right]=\mathbf{T}^{\prime} \mathbf{R}^{\prime}
$$

where $\mathbf{T}^{\prime}$ is an antisymmetric matrix and $\mathbf{R}^{\prime}$ is a rotation matrix. We have now:

$$
\sigma^{-1} \mathrm{UE}=\mathrm{F}=\mathbf{A F ^ { \prime }}=\mathbf{T}^{\prime} \mathbf{R}^{\prime} \mathbf{F}^{\prime}
$$

thus

$$
\mathbf{E}=\mathbf{U}^{\top}\left(\sigma \mathbf{T}^{\prime}\right) \mathbf{R}^{\prime} \mathbf{F}^{\prime}=\left(\mathbf{U}^{\top}\left(\sigma \mathbf{T}^{\prime}\right) \mathbf{U}\right)\left(\mathbf{U}^{\top} \mathbf{R}^{\prime} \mathbf{F}^{\prime}\right)
$$

'The result follows since $U^{\top}\left(\sigma T^{\prime}\right) U$ is antisymmetric, and $U^{\top} \mathbf{R}^{\prime} F^{\prime}$ is a rotation matrix.

We derive the conditions on the coefficients of $\mathbf{E E}^{\top}$ that imply (and are implied by) the equality of its two nonzero singular values. We define the coefficients $a, b, c$ of the characteristic polynomial of $\mathbf{E E}^{\top}$ by

$$
\operatorname{det}\left(\mathbf{E} \mathbf{E}^{\top}-\lambda \mathbf{I}\right)=-\lambda^{3}+a \lambda^{2}+b \lambda+c
$$

Since $c=\operatorname{det}\left(\mathbf{E E}^{\top}\right)=\operatorname{det}^{2}(\mathbf{E})$, we have $c=0$. Therefore the conclition we seek is eguivalent to witing that the quadratic equation $-\lambda^{2}+a \lambda+b$ has two equal roots:

$$
a^{2}+4 b=0
$$


If we define $a_{i}, b_{i}$ by

$$
\mathrm{EE}^{\top}=\left[\begin{array}{lll}
a_{1} & b_{3} & b_{2} \\
b_{3} & a_{2} & b_{1} \\
b_{2} & b_{1} & a_{3}
\end{array}\right]
$$

then it is easy to show that:

$$
\begin{aligned}
& a=a_{1}+a_{2}+a_{3} \\
& b=b_{1}^{2}+b_{2}^{2}+b_{3}^{2}-a_{1} a_{2}-a_{2} a_{3}-a_{3} a_{1}
\end{aligned}
$$

therefore:

$$
a^{2}+4 b=0=\left(a_{1}+a_{2}+a_{3}\right)^{2}+4\left(b_{1}^{2}+b_{2}^{2}+b_{3}^{2}-a_{1} a_{2}-a_{2} a_{3}-a_{3} a_{1}\right)
$$

We combine (6) and Theorem 1 to obtain the following result.

Proposition 3 A $3 \times 3$ matrix $\mathbf{E}$ with row vectors $\mathbf{e}_{i}^{\top}$ can be decomposed as the product $\mathbf{T R}$ of an antisymmetric matrix $\mathbf{T}$ and a rotation matrix $\mathbf{R}$ if and only if

$$
\begin{aligned}
\mathbf{e}_{1}^{\top}\left(\mathbf{e}_{2} \wedge \mathbf{e}_{3}\right) & =0 \\
\left(\mathbf{e}_{1}^{2}+\mathbf{e}_{2}^{2}+\mathbf{e}_{3}^{2}\right)^{2} & =4\left(\left\|\mathbf{e}_{1} \wedge \mathbf{e}_{2}\right\|^{2}+\left\|\mathbf{e}_{2} \wedge \mathbf{e}_{3}\right\|^{2}+\left\|\mathbf{e}_{3} \wedge \mathbf{e}_{1}\right\|^{2}\right)
\end{aligned}
$$

Proof : Equation (7) is equivalent to $\operatorname{det}(\mathrm{E})=0$. On expressing the $a_{i}$ 's and the $b_{i}$ 's of (5) as functions of the $\mathbf{e}_{i}$ equation (6) yields

$$
\left(\mathbf{e}_{1}^{2}+\mathbf{e}_{2}^{2}+\mathbf{e}_{3}^{2}\right)^{2}+4\left(\left(\mathbf{e}_{2}^{\top} \mathbf{e}_{1}\right)^{2}+\left(\mathbf{e}_{3}^{\top} \mathbf{e}_{1}\right)^{2}+\left(\mathbf{e}_{1}^{\top} \mathbf{e}_{2}\right)^{2}-\mathbf{e}_{1}^{2} \mathbf{e}_{2}^{2}-\mathbf{e}_{2}^{2} \mathbf{e}_{3}^{2}-\mathbf{e}_{3}^{2} \mathbf{e}_{1}^{2}\right)=0
$$

from which (8) follows.

Even though Proposition 3 has a. nice interpretation in terms of the row vectors of matrix $\mathbf{E}$, it can also be expressed differently:

Proposition 4 A $3 \times 3$ matrix $\mathbf{E}$ with row vectors $\mathbf{e}_{i}^{\top}$ can be decomposed as the product $\mathbf{T R}$ of an antisymmetric matrix $\mathrm{T}$ and a rotation malrix $\mathrm{R}$ if and only if

$$
\begin{gathered}
\operatorname{det}(\mathbf{E})=0 \\
f(\mathbf{E}) \equiv \frac{1}{2} \operatorname{Tr}\left(\mathbf{E E}^{\top}\right)^{2}-\operatorname{Tr}\left(\left(\mathbf{E E}^{\top}\right)^{2}\right)=0
\end{gathered}
$$

Proof : The proof is simple; one notices that

$$
\operatorname{Tr}\left(\mathrm{EE}^{\top}\right)^{2}-2 \operatorname{Tr}\left(\left(\mathrm{EE}^{\top}\right)^{2}\right)=-\left(a^{2}+4 b\right)
$$




\section{Algebraic geometry approach}

It is clear from section 3 that the properties of essential matrices can be described by polynomial equations. Following this line, Demazure has used the powerful tools of algebraic geometry to study the manifold $\mathcal{M}$ of all essential matrices [Dem88]. Here $\mathcal{M}$ is regarded as a submanifold of $\mathcal{P}^{8}$ by identifying matrices that differ by a non-zero scalar multiple, thus $\mathcal{P}^{8}$ is a quotient of the 9 dimensional space of all $3 \times 3$ matrices. The manifold $\mathcal{M}$ is the range of an application which associates to each pair $(\mathbf{R}, \mathbf{t})$ the essential matrix $\mathbf{E}=\mathbf{T R}$. Demazure does not restrict $\mathbf{R}$ and $\mathbf{t}$ to be real; but $\mathbf{R}$ must be orthogonal, that is, $\mathbf{R R}^{\top}=\mathbf{I}$.

He shows that a rank 2 matrix is an essential matrix if and only if it satisfies a certain set of 9 homogeneous polynomial equations of degree 3 . He then shows that the intersection of $\mathcal{M}$ with a codimension 5 subspace of $\mathcal{P}^{8}$ contains exactly 10 distinct points (a subspace of codimension $n$ is one defined by $n$ linear constraints). It follows that $\mathcal{M}$ has degree 10 , and that there are, in general, exactly 10 essentially different camera motions compatible with 5 given image correspondences.

We derive the 9 equations defining $\mathcal{M}$ and obtain two interesting new results. The first one is the solution to a puzzle, the second one is a result on the number of ambiguous motions when a large number of matches is available.

\subsection{The equations of $\mathcal{M}$}

Using Proposition 2 we can write:

$$
\frac{1}{2} \operatorname{Tr}\left(\mathbf{E} \mathbf{E}^{\top}\right) \mathbf{I}-\mathbf{E} \mathbf{E}^{\top}=\mathbf{t} \mathbf{t}^{\top}
$$

We multiply on the right by $\mathrm{E}$ and use Proposition 1 to obtain:

$$
\frac{1}{2} \operatorname{Tr}\left(E^{\top}\right) E-E E^{\top} \mathbf{E}=0
$$

The components of (11) yjeld 9 homogeneous polynomial equations of degree 3 which must be satisfied by the coefficients of E. Conversely, any rank 2 matrix which satisfies (11) is an essential matrix [Dem88]. Demazure shows that the equations of (11) are linearly independent, and that they define the algebraic manifold $\mathcal{M}$ of essential matrices.

We remark that the condition $\operatorname{det}(\mathbf{E})=0$ follows from (11). Suppose, if possible, that $\mathbf{E}$ is a matrix satisfying (11), such that $\operatorname{det}(\mathbf{E}) \neq 0$. Then $\mathbf{E}$ is invertible, thus (11) yields $1 / 2 \operatorname{Tr}\left(\mathbf{E E}^{\top}\right) \mathbf{I}=\mathbf{E E}^{\top}$. It follows that $3 / 2 \operatorname{Tr}\left(\mathbf{E E}^{\top}\right)=\operatorname{Tr}\left(\mathbf{E} \mathbf{E}^{\top}\right)$, thus $\operatorname{Tr}\left(\mathbf{E E}^{\top}\right)=0$. On substituting $\operatorname{Tr}\left(\mathbf{E} \mathbf{E}^{\top}\right)=0$ into (11) and taking determinants we obtain $\operatorname{det}(\mathbf{E})^{3}=0$, contradicting the hypothesis that $\operatorname{det}(\mathbf{E}) \neq 0$.

\subsection{Resolving an apparent contradiction}

There is an apparent contradiction since in proposition 4 we characterized the real points of the manifold $\mathcal{M}$ with two real equations (9) and (10), and in (11) we have 9 equations which also characterize the real (as well as the complex) points of $\mathcal{M}$. This contradiction is resolved by demonstrating that a real matrix satisfies (11) if and only if it satisfies (9) and (10). We begin with a definition. Let

$$
\mathbf{F}(\mathbf{E}) \equiv \frac{1}{2} \operatorname{Tr}\left(\mathbf{E} \mathbf{E}^{\top}\right) \mathbf{E}-\mathbf{E E}^{\top} \mathbf{E}
$$
We note that $\operatorname{Tr}\left(\mathbf{F}(\mathbf{E}) \mathbf{F}(\mathbf{E})^{\top}\right)$ is the sum of the squares of the entries of the left-hand side of (11). We show
that:

$$
\operatorname{Tr}\left(\mathbf{F}(\mathbf{E}) \mathbf{F}(\mathbf{E})^{\top}\right)=-\frac{1}{2} \operatorname{Tr}\left(\mathbf{E} \mathbf{E}^{\top}\right) \operatorname{Tr}\left(\mathbf{F}(\mathbf{E}) \mathbf{E}^{\top}\right)+3 \operatorname{det}(\mathbf{E})^{2}
$$


We write:

$$
\mathbf{F}(\mathbf{E})=\left(\frac{1}{2} \operatorname{Tr}\left(\mathbf{E} \mathbf{E}^{\top}\right) \mathbf{I}-\mathbf{E E}^{\top}\right) \mathbf{E}
$$

Letting $\mathbf{A}=\mathbf{E E}^{\top}$, we can write two equations:

$$
\begin{aligned}
\mathbf{F}(\mathbf{E}) \mathbf{E}^{\top} & =\left(\frac{1}{2} \operatorname{Tr}(\mathbf{A})-\mathbf{A}\right) \mathbf{A} \\
\mathbf{F}(\mathbf{E}) \mathbf{F}(\mathbf{E})^{\top} & =\frac{1}{4} \operatorname{Tr}(\mathbf{A})^{2} \mathbf{A}-\operatorname{Tr}(\mathbf{A}) \mathbf{A}^{2}+\mathbf{A}^{3}
\end{aligned}
$$

Taking the trace of the first equation, we obtain:

$$
\operatorname{Tr}\left(\mathbf{F}(\mathbf{E}) \mathbf{E}^{\top}\right)=\frac{1}{2} \tau_{1}^{2}-\tau_{2}
$$

where $\tau_{1}=\operatorname{Tr}(\mathbf{A}), \tau_{2}=\operatorname{Tr}\left(\mathbf{A}^{2}\right)$. Taking the trace of the second equation, we obtain:

$$
\operatorname{Tr}\left(\mathbf{F}(\mathbf{E}) \mathbf{F}(\mathbf{E})^{\top}\right)=\frac{1}{4} \tau_{1}^{3}-\tau_{1} \tau_{2}+\tau_{3}
$$

where $\tau_{3}=\operatorname{Tr}\left(\mathbf{A}^{3}\right)$. We recall Newton's formula:

$$
\tau_{3}=-\frac{1}{2} \tau_{1}^{3}+\frac{3}{2} \tau_{1} \tau_{2}+3 \operatorname{det}(\mathbf{A})
$$

On combining equations (13), (14), (15), we obtain (12).

It follows from (12) and the definition: of $f(\mathbf{E})$ given in (10) that

$$
\operatorname{Tr}\left(\mathbf{F}(\mathbf{E}) \mathbf{F}(\mathbf{E})^{\top}\right)=-\frac{1}{2} \operatorname{Tr}\left(\mathbf{E} \mathbf{E}^{\top}\right) f(\mathbf{E})+3 \operatorname{det}(\mathbf{E})^{2}
$$

If $\mathrm{E}$ is a real essential matrix, then $\mathrm{F}(\mathrm{E})=0, \operatorname{det}(\mathrm{E})=0$, and therefore $f(\mathrm{E})=0$. Reciprocally, if $f(\mathbf{E})=\operatorname{det}(\mathbf{E})=0$ and $\mathrm{E}$ is real, we have $\sum F_{i j}(\mathbf{E})^{2}=0$. Since the $F_{i j}$ 's are real, each one of them. is equal to 0 and $\mathbf{F}(\mathbf{E})=0$, therefore $\mathbf{E}$ is an essential matrix [Dem88]. This resolves the contradiction.

\subsection{Properties of $\mathcal{M}$}

It can be shown that the dimension of the manjfold $\mathcal{M}$ of essential matrices is 5 , since an essential matrix depends on five parameters, three arising from the rotation and two arising from the direction of the translation. The magnitude of the translation does not appear because it contributes only a scale factor, which has no effect since $\mathcal{M}$ is embedded in $\mathcal{P}^{8}$.

The degree of $\mathcal{M}$ is harder to obtain. It is, by definition, the number of intersections of $\mathcal{M}$ with a linear manifold of codimension 5. A linear manifold of codimension 5 can be considered as the intersection of 5 hyperplanes with equations

$$
\mathrm{m}^{\top} \mathrm{E} \mathrm{m}^{\prime}=0
$$

Therefore, the degree of $\mathcal{M}$ is precisely the answer to the question of how many solutions we obtain from 5 . point matches, Demazure shows that the answer is 10 . We prove the same result with a different approach in section 5 .

We remark that not every hyperplane in $\mathcal{P}^{8}$ satisfies an equation like (16), however, each linear manifold of codimension 5 can be defined by the intersection of 5 hyperplanes defined by equations like (16), for appropriate choices of $\mathbf{m}$ and $\mathbf{m}^{\prime}$. This is because the number of degrees of freedom is 4 for each equation ( 2 each for $\mathbf{m}$ and $\mathbf{m}^{\prime}$ ), giving a total of 20 degrees of freedom. This is precisely the same as the number of degrees of freedom of a projective space of dimension 3 (codimension 5 ) in $\mathcal{P}^{8}$ [SR 49]. 


\subsection{Ten real solutions}

We can build on a result by Demazure to obtain sets of 10 real camera motions compatible with 5 image correspondences. We choose the points in the two retinas to be

$$
\begin{aligned}
& a=\mathbf{a}^{\prime}=\mathbf{f}_{1} \\
& \mathbf{b}=\mathbf{b}^{\prime}=\mathbf{f}_{2} \\
& \mathbf{c}=\mathbf{c}^{\prime}=\mathbf{f}_{3} \\
& \mathbf{d}=\mathbf{e}^{\prime} \\
& \mathbf{e}=\mathbf{d}^{\prime}
\end{aligned}
$$

where the vectors $f_{i}$ form a standard basis. Then, according to Demazure ([Dem88]), there are 10 real camera motions compatible with these pairs. Although this example provides 10 distinct real camera motions, many of the reconstructed points are at infinity. This is because the matched points are very symmetric. But we can argue that by continuity, if we perturb the coordinates of the retina points "slightly", we should still find 10 distinct real solutions with the possibility that none of the reconstructed points are at infinity. Indeed, we have been able to generate an arbitrarily large number of 10 -tuples of solutions by slightly perturbing the points of (17). An example is given in section 6.4.

\subsection{Degenerate cases}

A very interesting result that can be obtained using essential matrices is that of Longuet-Higgins, Maybank, and Horn [Lon87,May85,Hor87]. The question they solved is that of determining how many different motions (essential matrices) were possible given an arbitrary number of point matches. This is the same as asking how many essential matrices $\mathbf{E}_{i}$ can satisfy:

$$
\mathbf{m}^{\top} \mathbf{E}_{i} \mathbf{m}^{\prime}=0
$$

for pairs $\mathbf{m} \leftrightarrow \mathbf{m}^{\prime}$ where $\mathbf{m}, \mathbf{m}^{\prime}$ range over open subsets of the retinal plane. Our solution requires the following two propositions.

Proposition 5 Let $\mathrm{E}$ be an essential matrix and let $\mathrm{B}$ be an arbitrary matrix. Then $\mathrm{Em} \wedge \mathrm{Bm}=0$ for all $\mathrm{m}$ if and only if $\mathrm{B}=\lambda \mathrm{E}$ for some scalar $\lambda$.

Proof: If $\mathbf{B}=\lambda \mathbf{E}$ then $\mathbf{E} \cdot \mathbf{m} \wedge \mathbf{B m}=0$ for all $\mathbf{m}$. Conversel $y$, let $\mathbf{E}=\mathbf{T R}$. Then

$$
E m \wedge B m=\left(m^{\top} B^{\top} t\right) R m-\left(m^{\top} B^{T} R m\right) t=0
$$

for all $\mathbf{m}$. The vectors $\mathbf{R m}$ and $\mathbf{t}$ are, in general, linearly independent thus, for all $\mathbf{m}$,

$$
\mathbf{m}^{\top} \mathbf{B}^{\top} \mathbf{t}=\mathbf{0} \quad \text { and } \quad \mathbf{m}^{\top} \mathbf{B}^{\top} \mathbf{R m}=\mathbf{0}
$$

hence $\mathbf{B}^{\top} \mathbf{t}=0$, and $\mathbf{B}^{\top} \mathbf{R}$ is antisymmetric. It follows that $\mathbf{R}^{\top} \mathbf{B}$ is antisymmetric, thus $\mathbf{R}\left(\mathbf{R}^{\top} \mathbf{B}\right) \mathbf{R}^{\top}=\mathbf{B R}^{\top}$ is antisymmetric. Let $B=T^{\prime} R$, where $T^{\prime}$ is antisymmetric. Then

$$
t^{\top} T^{\prime} R=t^{\top} B=\left(B^{\top} t\right)^{\top}=0
$$

We obtain $\mathbf{t}^{\top} \mathbf{T}^{\prime}=0$, hence $\mathbf{T}^{\prime}=\lambda \mathbf{T}$ for some scalar $\lambda$. The result follows.

Proposition 6 Let $\mathbf{E}_{1}, \mathbf{E}_{2}, \mathbf{E}_{3}$ be essential matrices such that $\mathbf{m}^{\top} \mathbf{E}_{i} \mathbf{m}^{\prime}=0$ for $i=1,2,3$, where $\mathbf{m}-\mathbf{m}^{\prime}$, and $\mathbf{m}, \mathbf{m}^{\prime}$ both range over open subsets of $\mathcal{P}^{2}$. Then $\mathbf{E}_{1}, \mathbf{E}_{2}, \mathbf{E}_{3}$ are linearly dependent. 
Proof : The result holds if two of the $\mathbf{E}_{i}$ are dependent, thus we suppose that no two of the $\mathbf{E}_{i}$ are dependent. Define a transformation $\Sigma$ between open subsets of $\mathcal{P}^{2}$ by $\Sigma \mathbf{m}^{\prime}=\mathbf{m}$. The points $\mathbf{E}_{1} \mathbf{m}^{\prime}, \mathbf{E}_{2} \mathbf{m}^{\prime}$, $\mathbf{E}_{3} \mathbf{m}^{\prime}$ are orthogonal to $\mathbf{m}$, and by proposition 5 no two of $\mathbf{E}_{1} \mathbf{m}^{\prime}, \mathbf{E}_{2} \mathbf{m}^{\prime}, \mathbf{E}_{3} \mathbf{m}^{\prime}$ are parallel, in general. We
thus obtain

$$
\Sigma \mathbf{m}^{\prime}=\mathbf{E}_{1} \mathbf{m}^{\prime} \wedge \mathbf{E}_{2} \mathbf{m}^{\prime}=\mathbf{E}_{1} \mathbf{m}^{\prime} \wedge \mathbf{E}_{3} \mathbf{m}^{\prime}
$$

for almost all $\mathbf{m}^{\prime}$, thus $\Sigma$ is defined by polynomial equations of degree 2 in $\mathbf{m}^{\prime}$. Suppose that the equations defining $\Sigma$ have a commen factor of degree 1 . Then $\mathbf{m} \leftrightarrow \mathbf{m}^{\prime}$ is linear, thus there exists an invertible matrix A such that $\mathbf{m}=\mathbf{A m}^{\prime}$. We obtain $\mathbf{m}^{\prime \top} \mathbf{A}^{\top} \mathbf{E}_{i} \mathbf{m}^{\prime}=0$ for all $\mathbf{m}^{\prime}$, thus the $\mathbf{A}^{\top} \mathbf{E}_{i}$ are antisymmetric. Let $\mathbf{A}^{\top} \mathbf{E}_{i}=\mathbf{T}_{i}$ for $i=1,2,3$, and let $t_{i}$ be the vector corresponding to the antisymmetric matrix $\mathbf{T}_{i}$.

Then $\Sigma$ is undefined at each $t_{i}$. By hypothesis, the equations defining $\Sigma$ have a common factor of degree 1. The $t_{i}$ are zeros of this common factor, thus they are collinear, and thus the $\mathbf{T}_{i}$ are linearly dependent. Hence the $\mathbf{E}_{i}$ are linearly dependent as required.

If the equations defining $\Sigma$ do not have a common factor of degree 1 then $\mathbf{m} \leftrightarrow \mathbf{m}^{\prime}$ is quadratic. It follows from the symmetry between $\mathbf{m}$ and $\mathbf{m}^{\prime}$ that $\Sigma$ has an inverse, thus $\Sigma$ is an example of a quadratic transformation, as defined in [SK52] (see also Section B). Let $\mathbf{n}^{\prime}$ be a fixed point of $\mathcal{P}^{2}$ such that $\Sigma$ is defined at $\mathbf{n}^{\prime}$. Then $\mathbf{E}_{1} \mathbf{n}^{\prime}, \mathbf{E}_{2} \mathbf{n}^{\prime}, \mathbf{E}_{3} \mathbf{n}^{\prime}$ are linearly dependent, thus there exist coefficients $a_{1}, a_{2}, a_{3}$, possibly depending on $\mathbf{n}^{\prime}$, such that

Define the matrix $\mathrm{G}$ by

$$
a_{1} \mathrm{E}_{1} \mathbf{n}^{\prime}+a_{2} \mathrm{E}_{2} \mathbf{n}^{\prime}+a_{3} \mathbf{E}_{3} \mathbf{n}^{\prime}=0
$$

$$
\mathbf{G} \equiv a_{1} \mathbf{E}_{1}+a_{2} \mathbf{E}_{2}+a_{3} \mathbf{E}_{3}
$$

and define a transformation $\Phi$ from $\mathcal{P}^{2}$ to $\mathcal{P}^{2}$ by

$$
\Phi \mathrm{m}^{\prime}=\mathrm{E}_{1} \mathrm{~m}^{\prime} \wedge \mathrm{Gm}^{\prime}=\mathrm{E}_{1} \mathbf{m}^{\prime} \wedge\left(a_{2} \mathrm{E}_{2}+a_{3} \mathbf{E}_{3}\right) \mathbf{m}^{\prime}
$$

The vectors $E_{1} \mathbf{m}^{\prime} \wedge \mathbf{E}_{2} \mathbf{m}^{\prime}$ and $\mathbf{E}_{1} \mathbf{m}^{\prime} \wedge \mathbf{E}_{3} \mathbf{m}^{\prime}$ are parallel, thus either $\Phi=\Sigma$ or $\Phi=0$. The case $\Phi=\Sigma$ does not apply since $\Phi \mathbf{n}^{\prime}=0, \Sigma \mathbf{n}^{\prime} \neq 0$, thus $\Phi=0$. It follows that $\mathbf{E}_{1} \mathbf{m}^{\prime}$ and $\left(a_{2} \mathbf{E}_{2}+a_{3} \mathbf{E}_{3}\right) \mathbf{m}^{\prime}$ are parallel for all $\mathbf{m}^{\prime}$, thus by proposition $5, \mathrm{E}_{1}$ and $a_{2} \mathrm{E}_{2}+a_{3} \mathrm{E}_{3}$ are linearly dependent.

It is a consequence of Proposition 6 that the question of determining the maximum number of essential matrices compatible with a given set of image correspondences is equivalent to asking how many in tersection points a general line can have with $\mathcal{M}$. Since we saw that the equations of $\mathcal{M}$ are of degree 3 , the number of intersections of a line with $\mathcal{M}$ is at most 3 , which corresponds to the result obtained by the previous
authors.

\section{$5 \quad$ Projective geometry approach}

We now describe the second and older approach to C'hasles' problem incorporating the rigidity constraint. This problem was solved in 1913 by Erwin Kruppa. [Krn13] who found that there were in gencral at most 11 solutions, real or complex. This appears to contradict Demazure's result, given in section 4, who proved that the number of solutions is 10 . In what follows we derive Kruppa's equations and show that in fact there are in general 10 solutions. The interest of Kruppa's method as compared to Demazure's is that it. allows us to construct the solutions explicitly.

Let $\mathbf{a} \leftrightarrow \mathbf{a}^{\prime}, \mathbf{b} \leftrightarrow \mathbf{b}^{\prime}, \mathbf{c} \rightarrow \mathbf{c}^{\prime}, \mathbf{d} \leftrightarrow \mathbf{d}^{\prime}, \mathbf{e}-\mathbf{e}^{\prime}$ be 5 pairs of corresponding image points, and let $\mathbf{x}, \mathbf{x}^{\prime}$ be the unknown epipoles. Let $l_{a}, l_{b}, \ldots$ be the epipolar lines $\langle\mathbf{x}, \mathbf{a}\rangle,\langle\mathbf{x}, \mathbf{b}\rangle, \ldots$. After a change of projective coordinates, we can always consider that the points $\mathbf{a}, \mathbf{b}, \mathbf{c}, \mathbf{d}$ have coordinates:

$$
a=\left[\begin{array}{l}
1 \\
0 \\
0
\end{array}\right] \quad b=\left[\begin{array}{l}
0 \\
1 \\
0
\end{array}\right] \quad c=\left[\begin{array}{l}
0 \\
0 \\
1
\end{array}\right] \quad d=\left[\begin{array}{l}
1 \\
1 \\
1
\end{array}\right]
$$




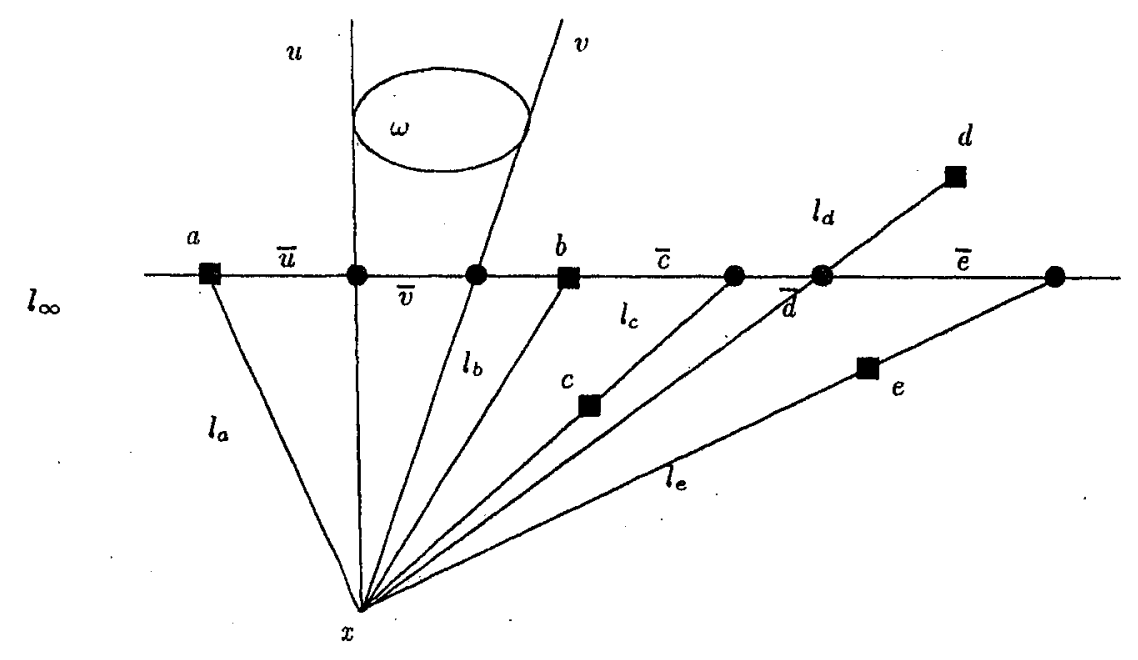

Figure 5: The situation in the image plane at the first time instant

Let the image $\omega$ of the absolute conic in the first retinal plane $\Pi$ (see appendix $B$ ) have the equation:

$$
\sum_{i=1}^{3} a_{i k} y_{i} y_{k}=0 \quad \text { with } \quad a_{i k}=a_{k i}
$$

We use the same notation for the second retinal plane $\Pi$ ' by adding ' whenever required.

The iclea is to consider the intersections of the epipolar lines with the lines $\langle\mathbf{a}, \mathbf{b}\rangle$ and $\left\langle\mathbf{a}^{\prime}, \mathbf{b}^{\prime}\right\rangle$, and to write down the conditions that these intersections are homographically related. In effect, $\langle\mathbf{a}, \mathrm{b}\rangle$, $\left\langle\mathbf{a}^{\prime}, \mathbf{b}^{\prime}\right\rangle$ provide a convenient set of coordinates for the two pencils of epipolar lines. We know 7 epipolar lines in each retinal plane, 5 from the image points and 2 from the tangents to the image of the absolute conic.

\subsection{Intersections of the epipolar lines with $\langle a, b\rangle$}

Let the epipolar lines $l_{a}, l_{b}, l_{c}, l_{d}, l_{e}$ intersect $\langle\mathbf{a}, \mathbf{b}\rangle$ at $\mathbf{a}, \mathbf{b}, \overline{\mathbf{c}}, \overline{\mathbf{d}}, \overline{\mathbf{e}}$ respectively, as depicted in figure 5. The intersections $\bar{c}, \bar{d}, \bar{e}$ of the epipolar lines $\langle\mathbf{x}, \mathbf{c}\rangle,\langle\mathbf{x}, \mathbf{d}\rangle,\langle\mathbf{x}, \mathbf{e}\rangle$ with the line at infinity $\langle\mathbf{a}, \mathbf{b}\rangle . \overline{\mathbf{u}}$ and $\overline{\mathrm{v}}$ are the intersections of the tangents $u$ and $v$ drawn from the epipole $\mathbf{x}$ to the image $\omega$ of the absolute conic $\Omega$.

The coordinates of $\mathbf{a}, \mathbf{b}, \overline{\mathbf{c}}, \overline{\mathbf{d}}, \overline{\mathbf{e}}$ as points of $\langle\mathbf{a}, \mathbf{b}\rangle$ are given by

$$
\begin{array}{cc}
\mathbf{a}=(1,0)^{\top} & \mathbf{b}=(0,1)^{\top} \\
\overline{\mathbf{c}}=\left(x_{1}, x_{2}\right)^{\top} & \overline{\mathbf{d}}=\left(x_{1}-x_{3}, x_{2}-x_{3}\right)^{\top} \\
\overline{\mathbf{e}}=\left(x_{1} e_{3}-x_{3} e_{1}, x_{2} e_{3}-x_{3} e_{2}\right)^{\top} &
\end{array}
$$

In order to find the intersection with $\langle\mathbf{a}, \mathbf{b}\rangle$ of the two tangents $u$ and $v$ to $\omega$ drawn from $\mathbf{x}$ we need the following result. Suppose we are given a conic $\omega$ with equation $\mathbf{y}^{\top} \mathbf{A y}=0$. Then the line $\langle\mathbf{x}, \mathbf{y}\rangle$ joining any two points $\mathrm{x}, \mathrm{y}$ is tangent to $\omega$ if and only if [SK52, chapter 5]:

$$
\left(\mathbf{x}^{\top} \mathrm{Ay}\right)^{2}=\left(\mathbf{x}^{\top} \mathrm{Ax}\right)\left(\mathbf{y}^{\top} \mathrm{Ay}\right)
$$




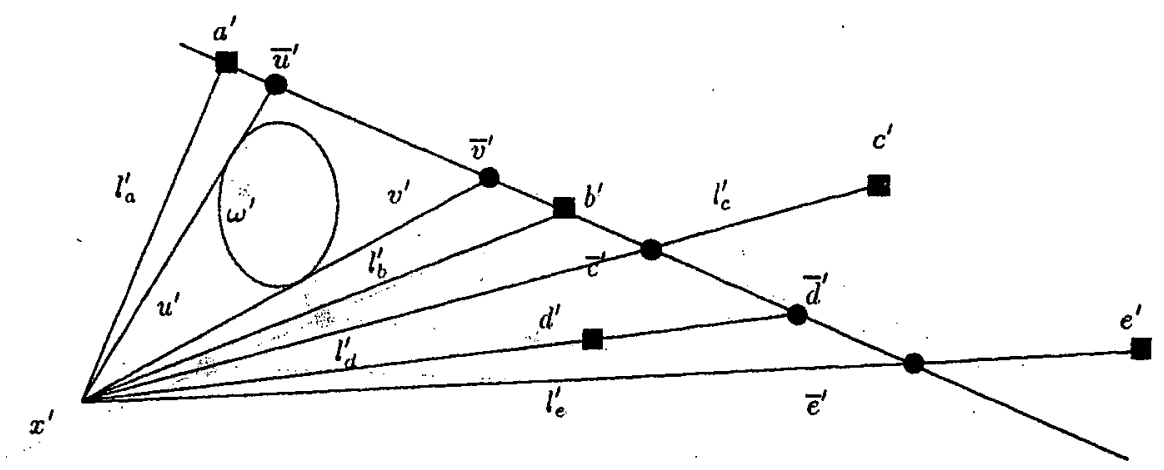

Figure 6: The situation in the image plane at the second time instant

It follows from (21), after some: algebra, that the coordinates $y_{1}, y_{2}$ on $<\mathbf{a}, \mathbf{b}>$ of the intersections $\overline{\mathbf{u}}$ and $\overline{\mathbf{v}}$ of the tangents $u$ and $v$ from: $\mathbf{x} \cdot$ to $\omega$ satisfy the following equation:

$$
A_{11} y_{1}^{2}+2 A_{12} y_{1} y_{2}+A_{22} y_{2}^{2}=0
$$

in which the coefficients $A_{i j}$ are quadratic functions of $\mathrm{x}$ :

$$
\begin{aligned}
& A_{11}=\delta_{12} x_{2}^{2}+\delta_{31} x_{3}^{2}+2 \delta_{1} x_{2} x_{3} \\
& A_{22}=\delta_{12} x_{1}^{2}+\delta_{23} x_{3}^{2}+2 \delta_{2} x_{1} x_{3} \\
& A_{12}=\delta_{3} x_{3}^{2}-\delta_{12} x_{1} x_{2}-\delta_{1} x_{1} x_{3}-\delta_{2} x_{2} x_{3}
\end{aligned}
$$

and the coefficients $\delta_{i}$ and $\delta_{i j}$ are quadratic functions of the $a_{i j}$. Specifically,

$$
\delta_{1}=a_{12} a_{13}-a_{11} a_{23}
$$

with $\delta_{2}$ and $\delta_{3}$ obtained by circular permutations of the indices; and

$$
\delta_{12}=a_{12}^{2}-a_{11} a_{22}
$$

with $\delta_{23}$ and $\delta_{31}$ obtained by circular permutations of the indices.

We now have to express that there exists a homography between $\langle\mathbf{a}, \mathbf{b}\rangle$ and $\left\langle\mathbf{a}^{\prime}, \mathbf{b}^{\prime}\right\rangle$ such that a $\bar{\Lambda} \cdot \mathbf{a}^{\prime}, \mathbf{b} \bar{\Lambda} \mathbf{b}^{\prime}, \overline{\mathbf{c}} \bar{\Lambda} \overline{\mathbf{c}}^{\prime}, \overline{\mathbf{d}}, \bar{\Lambda} \overline{\mathbf{d}}^{\prime}, \overline{\mathbf{e}} \bar{\Lambda} \overline{\mathbf{e}}^{\prime}$, and $(\overline{\mathbf{u}}, \overline{\mathbf{v}}) \bar{\Lambda}\left(\overline{\mathbf{u}}^{\prime}, \overline{\mathbf{v}}^{\prime}\right)$. Since a homography on a projective line is determined by three point correspondences and since we have seven, we obtain four equations. Since we have four unknowns, two for the (projective) coordinates of $\mathbf{x}$ and two for those of $\mathbf{x}^{\prime}$ we should be able to solve the problem. The situation in: the second image plane is shown in figure 6 . The intersections $\overline{\mathbf{c}}^{\prime}, \overline{\mathrm{d}}^{\prime}, \overline{\mathrm{e}}^{\prime}$ of the epipolar lines $\left\langle\mathbf{x}^{\prime}, \mathbf{c}^{\prime}\right\rangle,\left\langle\mathbf{x}^{\prime}, \mathbf{d}^{\prime}\right\rangle,\left\langle\mathbf{x}^{\prime}, \mathbf{e}^{\prime}\right\rangle$ with the line at infinity $\left\langle\mathbf{a}^{\prime}, \mathbf{b}^{\prime}\right\rangle . \bar{u}^{\prime}$ and $\bar{v}^{\prime}$ are the intersections of the tangents $u^{\prime}$ and $v^{\prime}$ drawn from the epipole $\mathrm{x}^{\prime}$ to the image $\omega^{\prime}$ of the absolute conic $\Omega$.

\subsection{Writing the equations}

The homographic correspondence $H$ between $<a, b\rangle$ and $\left\langle a^{\prime}, b^{\prime}\right\rangle$ satisfies $\mathbf{a} \pi \mathbf{a}^{\prime}$ and $\mathbf{b} \pi \mathbf{b}^{\prime}$ therefore it has a very simple form:

$$
\left\{\begin{array}{l}
\rho y_{1}^{\prime}=\alpha y_{1} \\
\rho y_{2}^{\prime}=\beta y_{2}
\end{array}\right.
$$


where $\alpha, \beta, \rho$ are nonzero unknowns. We rewrite this equation as:

$$
\rho \mathbf{y}^{\prime}=\left[\begin{array}{ll}
\alpha & 0 \\
0 & \beta
\end{array}\right] \mathbf{y}=\mathrm{Hy}
$$

Applying this to the points $\overline{\mathbf{c}}, \overline{\mathbf{d}}$, and $\overline{\mathbf{e}}$ we have:

$$
\begin{gathered}
\left\{\begin{array} { l } 
{ \lambda x _ { 1 } ^ { \prime } = \alpha x _ { 1 } } \\
{ \lambda x _ { 2 } ^ { \prime } = \beta x _ { 2 } }
\end{array} \quad \left\{\begin{array}{l}
\mu\left(x_{1}^{\prime}-x_{3}^{\prime}\right)=\alpha\left(x_{1}-x_{3}\right) \\
\mu\left(x_{2}^{\prime}-x_{3}^{\prime}\right)=\beta\left(x_{2}-x_{3}\right)
\end{array}\right.\right. \\
\left\{\begin{array}{l}
\nu\left(x_{1}^{\prime} e_{3}^{\prime}-x_{3}^{\prime} e_{1}^{\prime}\right)=\alpha\left(x_{1} e_{3}-x_{3} e_{1}\right) \\
\nu\left(x_{2}^{\prime} e_{3}^{\prime}-x_{3}^{\prime} e_{2}^{\prime}\right)=\beta\left(x_{2} e_{3}-x_{3} e_{2}\right)
\end{array}\right.
\end{gathered}
$$

Where $\lambda, \mu, \nu$ are nonzero unknowns. Taking ratios we eliminate $\alpha, \beta, \lambda, \mu$ and $\nu$ :

$$
\begin{gathered}
\frac{x_{1}^{\prime}-x_{3}^{\prime}}{x_{2}^{\prime}-x_{3}^{\prime}} \times \frac{x_{2}^{\prime}}{x_{1}^{\prime}}=\frac{x_{1}-x_{3}}{x_{2}-x_{3}} \times \frac{x_{2}}{x_{1}} \\
\frac{x_{1}^{\prime} e_{3}^{\prime}-x_{3}^{\prime} e_{1}^{\prime}}{x_{2}^{\prime} e_{3}^{\prime}-x_{3}^{\prime} e_{2}^{\prime}} \times \frac{x_{2}^{\prime}}{x_{1}^{\prime}}=\frac{x_{1} e_{3}-x_{3} e_{1}}{x_{2} e_{3}-x_{3} e_{2}} \times \frac{x_{2}}{x_{1}}
\end{gathered}
$$

Applying $\mathbf{H}$ to the pairs $(\overline{\mathbf{u}}, \overline{\mathbf{v}})$ and $\left(\overline{\mathbf{u}}^{\prime}, \overline{\mathbf{v}}^{\prime}\right)$ we start from:

$$
A_{11}^{\prime} y_{1}^{\prime 2}+2 A_{12}^{\prime} y_{1}^{\prime} y_{2}^{\prime}+A_{22}^{\prime} y_{2}^{\prime 2}=0
$$

Using the definition of $\mathbf{H}$ (equation (23)), we obtain:

$$
\alpha^{2} A_{11}^{\prime} y_{1}^{2}+2 \alpha \beta A_{12}^{\prime} y_{1} y_{2}+\beta^{2} A_{22}^{\prime} y_{2}^{2}=0
$$

which should have the same roots as equation (22). This implies that:

$$
\left\{\begin{array}{l}
\sigma A_{11}=\alpha^{2} A_{11}^{\prime} \\
\sigma A_{22}=\beta^{2} A_{22}^{\prime} \\
\sigma A_{12}=\alpha \beta A_{12}^{\prime}
\end{array}\right.
$$

Where $\sigma$ is a nonzero unknown. Agajn, we can eliminate $\sigma, \alpha$, and $\beta$ :

$$
\begin{aligned}
& \frac{A_{11}}{A_{12}} \times \frac{x_{1}}{x_{2}}=\frac{A_{11}^{\prime}}{A_{12}^{\prime}} \times \frac{x_{1}^{\prime}}{x_{2}^{\prime}} \\
& \frac{A_{22}}{A_{12}} \times \frac{x_{2}}{x_{1}}=\frac{A_{22}^{\prime}}{A_{12}^{\prime}} \times \frac{x_{2}^{\prime}}{x_{1}^{\prime}}
\end{aligned}
$$

We have thus obtained our four equations. In [Kru]3] Kruppa obtains all four equations directly from cross-ratios (see section B). Our derivation is longer, but otherwise exactly equivalent. 


\subsection{Simplifying the equations}

We can simplify equations (24) to (27) by applying the changes of variables $\Phi, \Phi^{\prime}$ defined by

$$
\begin{aligned}
\mathbf{x}=\Phi \mathbf{u} & \equiv\left(u_{2} u_{3}, u_{3} u_{1}, u_{1} u_{2}\right)^{\top} \\
\mathbf{x}^{\prime}=\Phi^{\prime} \mathbf{u}^{\prime} & \equiv\left(u_{2}^{\prime} u_{3}^{\prime}, u_{3}^{\prime} u_{1}^{\prime}, u_{1}^{\prime} u_{2}^{\prime}\right)^{\top}
\end{aligned}
$$

On applying $\Phi$ and $\Phi^{\prime}$ to (24) and (25) we obtain, after cancelling $u_{1} u_{2} u_{3}$ and $u_{1}^{\prime} u_{2}^{\prime} u_{3}^{\prime}$,

$$
\begin{gathered}
\frac{u_{3}-u_{1}}{u_{3}-u_{2}}=\frac{u_{3}^{\prime}-u_{1}^{\prime}}{u_{3}^{\prime}-u_{2}^{\prime}} \\
\frac{u_{3} e_{3}-u_{1} e_{1}}{u_{3} e_{3}-u_{2} e_{2}}=\frac{u_{3}^{\prime} e_{3}^{\prime}-u_{1}^{\prime} e_{1}^{\prime}}{u_{3}^{\prime} e_{3}^{\prime}-u_{2}^{\prime} e_{2}^{\prime}}
\end{gathered}
$$

On applying $\Phi$ and $\Phi^{\prime}$ to (26) we obtain, after cancelling $u_{1}^{2} u_{2} u_{3}$ and $u_{1}^{\prime 2} u_{2}^{\prime} u_{3}^{\prime}$,

$$
\begin{gathered}
\frac{\delta_{12} u_{3}^{2}+\delta_{13} u_{2}^{2}+2 \delta_{1} u_{3} u_{2}}{\delta_{3} u_{1} u_{2}-\delta_{12} u_{3}^{2}-\delta_{1} u_{2} u_{3}-\delta_{2} u_{1} u_{3}}= \\
\frac{\delta_{12}^{\prime} u_{3}^{\prime 2}+\delta_{13}^{\prime} u_{2}^{\prime 2}+2 \delta_{1}^{\prime} u_{3}^{\prime} u_{2}^{\prime}}{\delta_{3}^{\prime} u_{1}^{\prime} u_{2}^{\prime}-\delta_{12}^{\prime} u_{3}^{\prime 2}-\delta_{1}^{\prime} u_{2}^{\prime} u_{3}^{\prime}-\delta_{2}^{\prime} u_{1}^{\prime} u_{3}^{\prime}}
\end{gathered}
$$

On applying $\Phi$ and $\Phi^{\prime}$ to (27) and cancelling $u_{1} u_{2}^{2} u_{3}$ and $u_{1}^{\prime} u_{2}^{\prime 2} u_{3}^{\prime}$ we obtain

$$
\begin{gathered}
\frac{\delta_{12} u_{3}^{2}+\delta_{23} u_{1}^{2}+2 \delta_{2} u_{1} u_{3}}{\delta_{3} u_{1} u_{2}-\delta_{12} u_{3}^{2}-\delta_{1} u_{2} u_{3}-\delta_{2} u_{1} u_{3}} \\
\frac{\delta_{12}^{\prime} u_{3}^{\prime 2}+\delta_{23}^{\prime} u_{1}^{\prime 2}+2 \delta_{2}^{\prime} u_{1}^{\prime} u_{3}^{\prime}}{\delta_{3}^{\prime} u_{1}^{\prime} u_{2}^{\prime}-\delta_{12}^{\prime} u_{3}^{\prime 2}-\delta_{1}^{\prime} u_{2}^{\prime} u_{3}^{\prime}-\delta_{2}^{\prime} u_{1}^{\prime} u_{3}^{\prime}}
\end{gathered}
$$

\subsection{Finding the solutions in one retinal plane}

We must now find a way of eliminating $\mathbf{u}^{\prime}$ from (28) - (31). We notice that (28) and (29) together define a transformation $\Sigma$ from the plane $\left(u_{1}, u_{2}, u_{3}\right)$ to the plane $\left(u_{1}^{\prime}, u_{2}^{\prime}, u_{3}^{\prime}\right)$. After some algebra, it can be shown that $\Sigma$ is given by:

$$
\mathbf{u}^{\prime}=\Sigma \mathbf{u}=\left[\begin{array}{c}
e_{2}^{\prime}[e u]_{2}[1 u]_{3}-e_{3}^{\prime}[e u]_{3}[1 u]_{2} \\
e_{3}^{\prime}[e u]_{3}[1 u]_{1}-e_{1}^{\prime}[e u]_{1}[1 u]_{3} \\
e_{1}^{\prime}[e u]_{1}[1 u]_{2}-e_{2}^{\prime}[e u]_{2}[1 u]_{1}
\end{array}\right]
$$

in which we have used:

$$
[e u]_{1}=e_{2} u_{2}-e_{3} u_{3} \quad[1 u]_{1}=u_{2}-u_{3}
$$

The terms $[e u]_{2},[1 u]_{2}$ and $[e u]_{3},[1 u]_{3}$ are obtained by circular permutations. $\Sigma$, and also $\Phi$ and $\Phi^{\prime}$ are quadratic transformations; such transformations are the simplest generalization of the collineations (see section $B$ ).

If we now use (32) to substitute for $u_{1}^{\prime}, u_{2}^{\prime}, u_{3}^{\prime}$ in (30) and (31), we obtain two polynomial equations of degree 6 in $u_{1}, u_{2}, u_{3}$, which we denote by $A(\mathbf{u})=0, B(\mathbf{u})=0$. Each equation represents a sextic curve in the plane $\left(u_{1}, u_{2}, u_{3}\right)$, and the solutions for $\Phi(\mathbf{x})$ are among the points of intersection of these two sextics.

Algebraic geometry [SK52] tells us that the number of intersections of two algebraic curves of degrees $m$ and $n$ is $m n$, therefore we may have here as many as 36 solutions, real or complex. We are now going to see that not all these solutions are possible, and that 26 can be eliminated, leaving only 10 . 


\section{5 - Eliminating impossible solutions}

There are three sources of impossible solutions:

1. those which make the products $u_{1} u_{2} u_{3}$ and $u_{1}^{\prime} u_{2}^{\prime} u_{3}^{\prime}$ by which we simplified our equations to obtain equations (30) and (31) equal to 0.

2. the points $\mathbf{m}$ such that $\Sigma \mathrm{m}=(0,0,0)^{\top}$. Such points are called the fundamental points of $\Sigma$ ([SK52, Chapter 9] and section B).

3. if we denote by $D$ and $D^{\prime}$ the denominators in equations (30) and (31), then the points of intersection of the curves $D=0$ and $\Sigma^{-1}\left(D^{\prime}\right)=0$ are impossible solutions.

Let us consider each case in turn.

Case 1 In order for the product $u_{1} u_{2} u_{3}$ to be equal to 0 , at least one of the factors has to be equal to 0 . If we assume $u_{3}=0$, then we realize using for example a system for symbolic computation (in our case MAPLE) that in the equation of $A$, the coefficients of the terms in $u_{1}^{6}, u_{1}^{5}$, and $u_{1}^{4}$ are equal to zero. This indicates that the point $(1,0,0)$ is on $A$ and has order 3 (see appendix A1). In addition, the point $(0,1,0)$ is on $A$ with order 1 , since the coefficient of $u_{3}^{6}$ is zero. Similarly, it can be shown that $(1,0,0)$ is of order 1 and $(0,1,0)$ is of order 3 on $B$. It can also be verified that the point $(0,0,1)$ is of order 1 on both curves.

We thus have the following table:

$\begin{array}{cccc} & (1,0,0) & (0,1,0) & (0,0,1) \\ A & 3 & 1 & 1 \\ B & 1 & 3 & 1\end{array}$

These three points are included in the intersection of $A$ and $B$ but do not, in general, yield solutions to the motion problem. Counting the orders, we see that we have eliminated $3 \times 1+3 \times 1+1 \times 1=7$ solutions.

Case 2 We have to find the fundamental points of the quadratic transformation $\Sigma$. There are three such points in general [SK52, chapter 9] which we can easily discover.

Let us define two linear applications $\mathrm{X}$ and $\mathrm{Y}$ by:

$$
\mathbf{X} \mathbf{u}=\left[\begin{array}{l}
{[1 u]_{1}} \\
{[1 u]_{2}} \\
{[1 . u]_{3}}
\end{array}\right] \quad \mathbf{Y} \mathbf{u}=\left[\begin{array}{c}
e_{1}^{\prime}[e u]_{1} \\
e_{2}^{\prime}[e u]_{2} \\
e_{3}^{\prime}[e u]_{3}
\end{array}\right]
$$

The matrices of $\mathrm{X}, \mathrm{Y}$ are

$$
\mathrm{X}=\left[\begin{array}{ccc}
0 & 1 & -1 \\
-1 & 0 & 1 \\
1 & -1 & 0
\end{array}\right] \quad \mathrm{Y}=\left[\begin{array}{ccc}
0 & e_{1}^{\prime} e_{2} & -e_{1}^{\prime} e_{3} \\
-e_{2}^{\prime} e_{1} & 0 & e_{2}^{\prime} e_{3} \\
e_{3}^{\prime} e_{1} & -e_{3}^{\prime} e_{2} & 0
\end{array}\right]
$$

and we have:

$$
\Sigma \mathbf{u}=\mathbf{X} \mathbf{u} \wedge \mathrm{Yu}
$$

The fundamental points are therefore those points $\mathbf{u}$ such that $\mathrm{Yu}=0$ or $\mathrm{Xu}=0$ or such that $\mathrm{Xu}$ and $\mathrm{Yu}$ are parallel.

The case $\mathbf{X} \mathbf{u}=0$ corresponds to the fundamental point $\overline{\mathbf{d}}=(1,1,1)^{\top}$, the case $\mathbf{Y u}=0$ corresponds to the fundamental point $\left(1 / e_{1}, 1 / e_{2}, 1 / e_{3}\right)^{\top}$, and the third case is solved a.s follows. Let $\overline{\mathbf{p}}$ be a point such that

$$
\mathbf{X} \overline{\mathbf{p}}=\lambda \mathbf{Y} \overline{\mathbf{p}}
$$


This is equivalent to:

$$
(\mathrm{X}-\lambda \mathrm{Y}) \overline{\mathbf{p}}=0
$$

The values of $\lambda$ for which the determinant of $X-\lambda Y$ is equal to 0 are $\lambda=0$ (corresponding to $X$ ), $\lambda=\infty$ (corresponding to $Y$ ), and a third value, $\lambda_{0}$, which can be computed, for example using MAPLE. For this value, it can be verified that the rank of $X-\lambda_{0} Y$ is, in general, 2 and that $\bar{p}$ is represented by any vector in the null space. Notice that the coordinates of $\overline{\mathbf{p}}$ can be explicitely computed as functions of the coordinates of $\mathbf{e}$ and $\mathbf{e}^{\prime}$.

Using again the power of symbolic processing, it is easy to show that the three points $\overline{\mathbf{d}}, \overline{\mathbf{e}}$, and $\overline{\mathbf{p}}$ are points of order 2 on $A$ and $B$.

$\begin{array}{llll} & \bar{d} & \bar{e} & \bar{p} \\ A & 2 & 2 & 2 \\ B & 2 & 2 & 2\end{array}$

These three points are included in the intersection of $A$ and $B$, but they do not, in general, yield solutions to the motion problem. Counting the orders, we see that we have eliminated 12 solutions (this corresponds to the case of figure 10 ).

Case 3 The curve $D$ is a conic, and $\Sigma^{-1}\left(D^{\prime}\right)$ is a quartic in $\mathbf{u}$, since $D^{\prime}\left(\mathbf{u}^{\prime}\right)$ is of degree 2 in $\mathbf{u}^{\prime}$ and $\mathbf{u}^{\prime}=\Sigma \mathbf{u}$ is of degree 2 in $\mathbf{u}$. These two curves have in general 8 intersections among which are included, as can be verified, the points $(1,0,0)$ and $(0,1,0)$ which have already been removed in case 1$)$. Since each of the remaining 6 points is of order 1 on $A$ and $B$, we have obtained six more solutions to eliminate.

On adding the impossible solutions obtained in cases 1,2 and 3 we obtain a total of 25 , thus there remain. 11 intersections of $A$ and $B$ to consider.

\subsection{Kruppa's spurious solution}

It turns out that $A$ and $B$ not only intersect at $(0,0,1)$, but that they are tangential at $(0,0,1)$, since the coefficients of $u_{3}^{5}$ in $A(\mathbf{u})$ and $B(\mathbf{u})$ are equal. Again, this can be verified, in fact discovered would be the right word, using MAPLE. The equation of the common tangent at $(0,0,1)$ is the coefficient of $u_{3}^{5}$, a linear polynomial in $u_{1}$ and $u_{2}$.

It follow's that $(0,0,1)$ must count twice in the intersection and not once (this corresponds to the case of figure 9); this eliminates one further solution, thus the number of solutions is reduced from 36 to 10 , real or complex, in agreement with Demazure's result.

\section{Computer implementation of Kruppa's method}

We have an implementation of Kruppa's method in MAPLE that can find all the real rigid motions compatible with 5 given image correspondences. The implementation follows the structure of Kruppa's method closely, as illustrated in Figure $\tau$.

\subsection{The implementation}

We simulate a camera configuration such as the one represented in figure 1 . We choose 5 points $p_{i}$ in space and a rigid motion $\mathbf{R}, \mathbf{t}$. We then compute the image coordinates of the $\mathbf{p}_{i}$ in the two retinal planes, and apply a linear transformation to each retinal plane such that relations (20) are satisfied. We then find the equations of $\omega$ and $\omega^{\prime}$ in the transformed planes. We use the quadratic transformations $\Phi$ and $\Sigma \Phi^{\prime}$ as shown in Figure 3 to obtain the two sextic polynomial constraints on $\mathbf{u}, A=0, B=0$. 


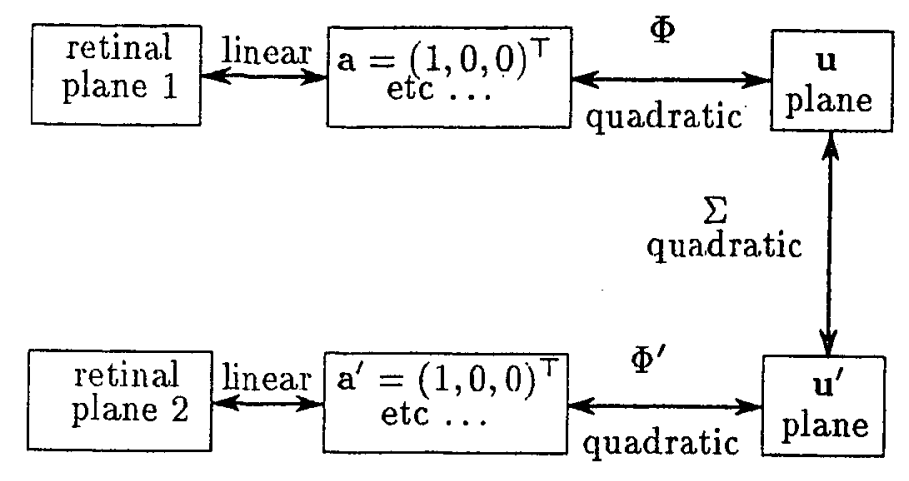

Figure 7: Block diagram of Kruppa's method

We eliminate $u_{3}$ from $A, B$ by computing the resultant, $R(A, B)$, which is a homogeneous polynomial in $u_{1}, u_{2}$ of degree 35 . Normally, $R(A, B)$ would have degree 36 . The degree is 35 in this case because $A$ and $B$ intersect at $(0,0,1)$.

The 35 roots of $R(A, B)$ correspond to the 35 intersections of $A$ and $B$ excluding $(0,0,1)$. As explained in section 5.5 , we know 26 of the intersections of $A, B$, hence we know 25 of the roots of $R(A, B)$. The factor of $R(A, B)$ corresponding to these roots is described in section 6.2. We divide out this factor of $R(A, B)$ to obtain a polynomial of degree 10 in $u_{1}, u_{2}, C^{\prime}$.

We find the 10 solutions of $C\left(u_{1}, u_{2}\right)=0$, and for each solution we find the associated value of $u_{3}$. Then $\Phi \mathbf{u}$ and $\Sigma \Phi^{\prime} \mathbf{u}$ yield the coordinates of the two epipoles in the transformed retinal planes. On applying linear transformations we obtain the epipoles $\mathbf{x}, \mathbf{x}^{\prime}$ in the original retinal planes. Each pair $\mathbf{x}, \mathbf{x}^{\prime}$ corresponds to a. unique essential matrix $E$ in $\mathcal{M}$ and $\mathbf{E}$ corresponds to a pair $\mathbf{R}, \mathbf{t}$ and $\mathbf{R}^{\prime}, \mathbf{t}$ of rigid motions in which $\mathbf{R}, \mathbf{R}^{\prime}$ differ by a rotation of $180^{\circ}$ about the axis $t$. We obtain $\mathbf{R}, \mathbf{t}$ and $\mathbf{R}^{\prime}, \mathbf{t}$ from $\mathbf{x}, \mathbf{x}^{\prime}$ using the method described in section 6.3 .

We check to see if each camera motion $R, t$ is physically feasible in that all the reconstructed points are in front of the camera in both positions. If the reconstructed points have coordinates $\mathrm{p}_{i}, \mathrm{p}_{i}^{\prime}$ with respect to the first and second camern positions respectively, then this is equivalent to showing that there exists a. vector $\mathbf{n}$ such that $\mathbf{n}^{\top} \mathbf{p}_{i}>0, \mathbf{n}^{\top} \mathbf{p}_{i}^{\prime}>0$ for $1 \leq i \leq 5$. The image plane can then be placed normal to $\mathbf{n}$. Feasibility is easily checked using the linear programming routines included in MAPLE.

We use rational numbers as far as possible, since this eliminates the rounding errors associated with real numbers. We are forced to switch to real numbers only in order to find the roots of $C\left(u_{1}, u_{2}\right)$. Here is an example of a polynomial $C\left(u_{1}, u_{2}\right)$ :

$-1449844079938203155299616438.5823829445312901164585110982089724065259581626379 u_{2}^{10}+$ $184691377803329029347748808309849217411128727619802792461137606190158844207633 u_{1} u_{2}^{9}+$ $16843332772422844573241514733119251525635414333777632963271483194648714284047009 u_{2}^{8} u_{1}^{2}-$ $24427098149339043141459891722203904911600557159986882512793434428682338733253738 u_{2}^{7} u_{1}^{3}+$ $63397105152646554594325265942738311478943741974390668059471392570469189409708382 u_{1}^{4} u_{2}^{6}-$ $66151900614213127592772967699668516337828351201756170648550776930882551225154496 u_{1}^{5} u_{2}^{5}+$ $29828529069911862072961630690477591478744475395915921663681143920847921768444928 u_{1}^{6} u_{2}^{4}-$ $3904560778519106865353130510371494328962126741245973466076501192834224843200512 u_{1}^{7} u_{2}^{3}-$ $808621896460637264694457561633024483115106410882501572723905296052700194564096 u_{1}^{8} u_{2}^{2}+$ $229346556720403191339947215738208465290131961576188304798361255541787487305728 u_{1}^{9} u_{2}-$ 


\section{$13141657256468899620669682070871427278101139616988962559826955187185776721920 u_{1}^{10}$}

It should be noted that all integer coefficients of the polynomial $C$ have no common divisor!

\begin{tabular}{|c|c|}
\hline Points in retina 1 & Points in retina 2 \\
\hline$a=\left[\begin{array}{lll}1000 & 2000 & 1000\end{array}\right]^{T}$ & $\mathbf{a}^{\prime}=\left[\begin{array}{ll}1100 & 1900\end{array}\right.$ \\
\hline$b=\left[\begin{array}{lll}1414 & -1414 & 1414\end{array}\right]^{T}$ & $\mathbf{b}^{\prime}=\left[\begin{array}{lll}1314 & -1514 & 1314\end{array}\right]^{T}$ \\
\hline$c=\left[\begin{array}{lll}-1732 & 0 & 1732\end{array}\right]^{T}$ & $c^{\prime}=\left[\begin{array}{lll}-1832 & 100 & 1632\end{array}\right]^{T}$ \\
\hline $\mathrm{d}=\left[\begin{array}{lll}2000 & 1000 & 3000\end{array}\right]^{T}$ & $\mathbf{d}^{\prime}=[-1100$ \\
\hline$e=\left[\begin{array}{lll}-1000 & -1000 & 2000\end{array}\right]^{T}$ & $\mathrm{e}^{\prime}=[2100$ \\
\hline
\end{tabular}

Table 1: Coordinates of matched points yielding 10 real solutions

\subsection{Factoring the resultant of $A$ and $B$}

We write $A$ and $B$ as polynomials in $u_{3}$ with coefficients $u_{i}, b_{i}$ that are polynomials of degree $i$ in: $u_{1}, u_{2}$. We obtain:

$$
\begin{aligned}
& A \equiv a_{1} u_{3}^{5}+a_{2} u_{3}^{4}+\ldots+a_{6} \\
& B \equiv b_{1} u_{3}^{5}+b_{2} u_{3}^{4}+\ldots+b_{6}
\end{aligned}
$$

The resultant $R(A, B)$ obtained by eliminating $u_{3}$ is a homogeneous polynomial in $u_{1}, u_{2}$ of degree 35 , the roots of which give the ratios of $u_{1}$ to $u_{2}$ at the intersections of $A$ and $B$, excluding the intersection at $(0,0,1)$. The resultant $R(A, B)$ contains a factor of degree 25 arising from the known intersections of $A$ and. $B$.

It follows from case 1) and case 2) of section 5.5 that $R(A, B)$ is divisible, as a polynomial in $u_{1}, u_{2}$, by ach of

$$
\begin{gathered}
u_{1}^{3} \\
u_{2}^{3} \\
\left(u_{1}-u_{2}\right)^{4} \\
\left(e_{1} u_{1}-e_{2} u_{2}\right)^{4} \\
\left(\bar{p}_{2} u_{1}-\bar{p}_{1} u_{2}\right)^{4}
\end{gathered}
$$

The factor of $R(A, B)$ arising from case 3$)$ of section 5.5 divides the resultant $R\left(D, \Sigma^{-1}\left(D^{\prime}\right)\right)$ obtained by eliminating $u_{3}$ from $D$ and $\Sigma^{-1}\left(D^{\prime}\right)$. We have

$$
\begin{aligned}
D & =c_{0} u_{3}^{2}+c_{1} u_{3}+c_{2} \\
\Sigma^{-1}\left(D^{\prime}\right) & =c_{0}^{\prime} u_{3}^{4}+c_{1}^{\prime} u_{3}^{3}+\ldots+c_{4}^{\prime}
\end{aligned}
$$


Thus $R\left(D, \Sigma^{-1}\left(D^{\prime}\right)\right)$ is a polynomial of degree 8 in $u_{1}, u_{2}$ which is divisible by $u_{1} u_{2}$, since $D$ and $\Sigma^{-1}\left(D^{\prime}\right)$ intersect at $(0,1,0)$ (where $\left.u_{1}=0\right)$, and at $(1,0,0)$ (where $\left.u_{2}=0\right)$. Define $Q$ by

$$
Q u_{1} u_{2}=R\left(D, \Sigma^{-1}\left(D^{\prime}\right)\right)
$$

Then $Q$ is a homogeneous polynomial of degree 6 in $u_{1}$ and $u_{2}$ which divides $R(A, B)$.

An additional factor of $R(A, B)$ arises from the tangency of $A$ and $B$ described in section 5.6. The coefficients $a_{1}, b_{1}$ are proportional, thus $R(A, B)$ is divisible by $a_{1}$.

On dividing $R(A, B)$ by all the above factors we are left with a polynomial of degree 10 in $u_{1}, u_{2}$, the roots of which yield the 10 solutions to the motion problem.

\subsection{Recovering rigid motion from the epipoles}

We have seen that Kruppa's method yields pairs of epipoles $\mathbf{x}, \mathbf{x}^{\prime}$ compatible with a given set of 5 image correspondences. In this section we describe a method for recovering the rigid motion $R, t$ from $x, x^{\prime}$. In addition to $\mathbf{x}, \mathbf{x}^{\prime}$ we require one pair $\mathbf{a} \rightarrow \mathbf{a}^{\prime}$ of corresponding points.

It follows from the definitions of $\mathbf{R}, \mathbf{t}$ and $\mathbf{x}, \mathbf{x}^{\prime}$ that

$$
\mathbf{x}=\mathbf{t} \quad \text { and } \quad \mathbf{x}^{\prime}=-\mathbf{R}^{\top} \mathbf{t}
$$

Let $\mathbf{S}$ be a rotation through $180^{\circ}$ such that $\mathbf{S t}=\mathbf{R}^{\top} \mathbf{t}$. To fix ideas, let the axis of $\mathbf{S}$ be the bisector of $t$ and $\mathbf{R}^{\top} \mathbf{t}$. Let $U=\mathbf{R S}$. It follows from the construction of $S$ that $U$ has a known axis $t$. The angle of rotation
$\theta$ of $U$ is unknown.

We have $\mathbf{a} \rightarrow \mathbf{a}^{\prime}$, thus $\mathbf{a}^{\prime \top} \mathbf{E} \mathbf{a}=0$, where $\mathbf{E}=\mathbf{T R}$ is the essential matrix associated with $\mathbf{R}, \mathrm{t}$. It follows that $\mathbf{a}^{\prime T}$ TRSSa $=0$, thus

The vectors $\mathbf{a}^{\prime T} \mathrm{~T}$ and $\mathrm{Sa}$ are both known, thus (33) yields an equation

$$
\left(\mathbf{a}^{\prime \top T} \mathbf{T}\right) \mathbf{U}(\mathrm{Sa})=0
$$

$$
\alpha x \cos (\theta)+\beta \sin (\theta)=0
$$

where $\alpha, \beta$ are known coefficients. There is no constant term in (34) because $\mathrm{a}^{\prime \top} \mathbf{T}$ is normal to the axis of $\mathrm{U}$. We solve (34) for $\theta$. Two solutions are obtained, differing lsy $180^{\circ}$, corresponding to the fact the second camera can be rotated in two ways about $t$. Having found $\theta$ and hence $U$, we recover $R$, using the equation
$\mathbf{R}=U$.

\subsection{Experimental results}

In our experiments we look for sets of 5 pairs of image correspondences with 10 real solutions for which none of the reconstructed points are al infinity. When referring to the number of solutions we always mean the number of pairs of epipoles or equivalently the number of essential matrices obtained.

We begin with a set of image correspondences $\mathbf{a}-\mathbf{a}^{\prime}, \mathbf{b} \rightarrow \mathbf{b}^{\prime}, \mathbf{c} \rightarrow \mathbf{c}^{\prime}, \mathbf{d} \rightarrow \mathbf{d}^{\prime}, \mathbf{e} \rightarrow \mathbf{e}^{\prime}$, in which $\mathbf{a}, \mathbf{b}$, $c$ and $\mathbf{a}^{\prime}, \mathbf{b}^{\prime}, \mathbf{c}^{\prime}$ are two triads of mutually orthogonal vectors, and $\mathbf{d}=\mathbf{e}^{\prime}, \mathbf{e}=\mathrm{d}^{\prime}$. As stated in section 4.4 , these image correspondences are known to be compatible with 10 distinct rigid motions. We then subject the image points to a small perturbation. If the perturbation is sufficiently small then we still have 10 real solutions, and if it is at the same time sufficiently general then none of the reconstructed points is at infinity.

We then check to see how many of the solutions are feasible. Each solution gives rise to two rigid motions $\mathbf{R}, \mathbf{t}$ and $\mathbf{R}^{\prime}, t$ in which $\mathbf{R}, \mathbf{R}^{\prime}$ differ by a rotation of $180^{\circ}$ about $t$. The solution is feasible if eitler $\mathbf{R}, \mathbf{t}$ or $\mathbf{R}^{\prime}, \mathbf{t}$ yjeld $3 \mathrm{D}$ reconstuctions in which all the points are in front of the camera for both cancra positions.

We give two examples of our results. In the first we have 10 real solutions, 3 of which are feasible and in the second we have 8 real solutions all of which are feasible. These results have been obtained from the 
points shown in tables 1 and 3. The points in table 1 correspond to a perturbed Demazure's configuration (see Section 4.4). Indeed, we have:

$$
\begin{array}{r}
a^{\top} b=a^{\top} c=b^{\top} c=0 \\
\mathbf{a}^{\top} a \approx b^{\top} b \approx c^{\top} c
\end{array}
$$

Also, $\mathbf{a}^{\prime}, \mathbf{b}^{\prime}, \mathbf{c}^{\prime}$ are almost equal to $\mathbf{a}, \mathbf{b}, \mathbf{c}$, respectively and:

$$
\begin{aligned}
& \mathrm{d} \approx \mathrm{e}^{\prime} \\
& \mathrm{e} \approx \mathbf{d}^{\prime}
\end{aligned}
$$

This configuration of points yields 10 distinct real solutions among which 3 are feasible (table 2).

In the second example, we apply a rigid motion to the camera (a 60.00145558 degrees rotation with respect to the $y_{3}$ axis, and a translation equal to $(0.01,0.01,-1)^{\top}$. The resulting points are shown in table 3. In this table, the coordinates of the points appear as real number, in fact the internal representation is rational to allow exact computions in the field of rationals. This is achieved by representing the rotation rationally through quaternions. Our MAPLE program yields 8 real distinct solutions, all of them feasible. The results are shown in table 4.

In both examples, the recovered $\mathbf{R}$ and $\mathbf{t}$ yield very small values (less than $10^{-6}$ ) of the error criterion:

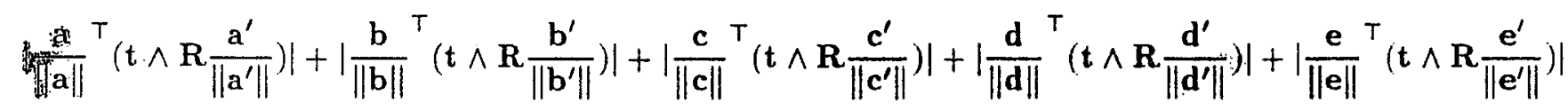

15i the second example, all 8 solutions have a rotation of roughly 60 degrees about roughly the $y_{3}$-axis, but differ in translation. The real solution is the 7 th.

\section{Conclusions}

We iave presented two very different approaches to the problem of finding the camera motions compatible with a given set of image correspondences, one method based on essential matrices and algebraic geometry, the wither method based on epipoles and projective geometry. Both approaches yield the result that there are, in general, exactly 10 camera motions compatible with 5 given image correspondences. The significance of the fignre 5 is that it is the least number of image correspondences compatible with only a finite number of camera motions. The figure 10 is a fundamental measure of the complexity of the problem of finding the camera motions, analogous to the degree of an algebraic curve. In this context 10 is high, indicating that, the problem is difficult.

We have seen that in certain cases the 10 solutions can all yield real valued rotations and translations, however, we have carried out many experiments with our implementation of Kruppa's method that yield only 2, 4, 6, or 8 real solutions. It appears that in many cases 4 real solutions are obtained, in line with the evidence of [Hor87]. Horn reports that the 4 real solutions often include only one feasible solution in whicil all the data points are in front of the camera. A concise and accessible description of the sets of image correspondences compatible with exactly one feasible camera motion would be of great interest, however such a description may be hard to obtain.

It, would also be of jnterest to explore more thoronghly the connections between the use of essential matrices and the use of epipoles for finding the camera motion. A notable feature of the second approach is that it is symmetric in the treatment of the two retinal planes, up to equations (30) and (31). In the first approach an asymmetry is introduced from the beginning since one must factor an essential matrix $\mathbf{E}$ either as $\mathrm{E}=\mathrm{TR}$ or as $\mathrm{E}=\mathrm{RT}$. 
The original motivation for this work is: to characterise the stability of the problem of finding camera motion. The results obtained so far suggest two possible sources of instability, depending on the form of the manifold $\mathcal{M}$ of essential matrices. The image correspondences determine linear subspaces of the 8 dimensional projective space containing the manifold $\mathcal{M}$ of essential matrices. Solutions to the motion problem then arise from the intersections of $\mathcal{M}$ with these linear subspaces. The fact that as many as 10 solutions can arise from 5 image correspondences suggests either that $\mathcal{M}$ has a complicated shape with high curvature leading to multiple intersections in "unexpected" places, or that $\mathcal{M}$ has low curvature and is "near parallel" to the linear subspaces. The currently available evidence leads us to favour the second view.

Stability and instability can also be considered in the context of Kruppa's method. The intersections of the two sextic plane curves may be unstable either because each sextic is individually unstable, or because the sextics are near parallel at their points of intersection.

\section{Acknowledgements}

We acknowledge the role of Thomas Buchanan who introduced us to the German literature on photogrammetry and motion and opened our eyes, as well as J.O. Eklundh, to the beauty of projective geometry. We also acknowledge the help of the MAPLE language for symbolic computation without which the experimental part of this work would have been totally impossible. The second author was employed by the Marcony Company during the writing of this paper. 


\section{References}

[Cha55] M. Chasles.

Question No. 296.

Nouv. Ann. Math., 14:50, 1855.

[Dem88] M. Demazure.

Sur deux problèmes de reconstruction.

Technical Report, INRIA, 1988.

Technical Report No. 882 .

[GVL83] G.H. Golub and C.F. Van Loan.

Matrix Computations.

Johns Hopkins University Press, 1983.

[Hes63] O. Hesse.

Die cubische Gleichung, von welcher die Losung des Problems der Homographie von M. Chasles abhangt.

J. reine angew. Math., 62:188-192, 1863.

[Hor87] B.K.P. Horn.

Closed-form Solution of Absolute Orientation using Unit Quaternions.

Journal of the Optical Society A, 4(4):629-642, April 1987.

[Kru13] E. Kruppa.

Zur Ermittlung eines Objektes aus zwei Perspektiven mit innerer Orientierung.

Sitz.-Ber. Akad. Wiss., Wien, math. naturw. Kl., Abt. IIa., 122:1939-1948, 1913.

[Lon81] H.C. Longuet-Higgins.

A computer algorithm for reconstructing a scene from two perspective projections.

Nalure, 293:133-135, 1981.

[Lon87] H.C. Longuet-Higgins.

Mental Processes: Studies in Cognitive Science, chapter Configurations that defeat the eight-point algorithm, pages 395-397.

MIT Press, Cambridge, MA-London, 1987.

[Lon88] H.C. Longuet-Higgins.

Multiple interpretations of a pair of images of a surface.

Proc. Roy. Soc. Lond. A., 1988.

[May85] S.J. Maybank.

The angular velocity associated with the optical flow field due to a rigid moving body.

Proc. Roy. Soc. Lond. A, 401:317-326, 1985.

[May87] S.J. Maybank.

A theoretical study of optical flow.

$\mathrm{PhD}$ thesis, University of London, Birkbeck College, November 1987.

[SK52] J.G. Semple and G.T. Kneebone.

Algebraic Projective Geometry. 
Oxford: Clarendon Press, 1952.

Reprinted 1979.

[SR49] J.G. Semple and L. Roth.

Introduction to Algebraic Geometry.

Oxford: Clarendon Press, 1949.

Reprinted 1987.

[Stu69] R. Sturm.

Das Problem der Projektivitat und seine Anwendung auf die Flachen zweiten Grades. Math. Ann., 1:533-574, 1869. 


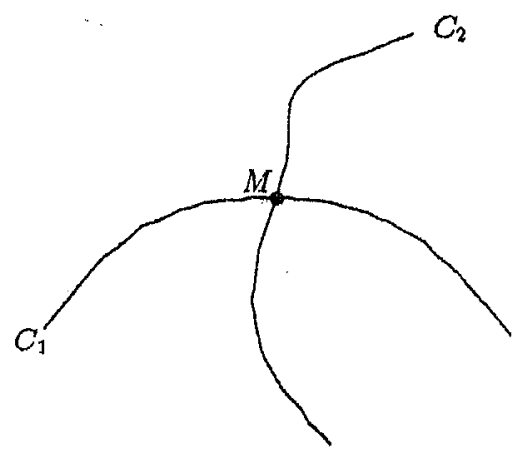

Figure 8: Intersection of two curves at a regular point

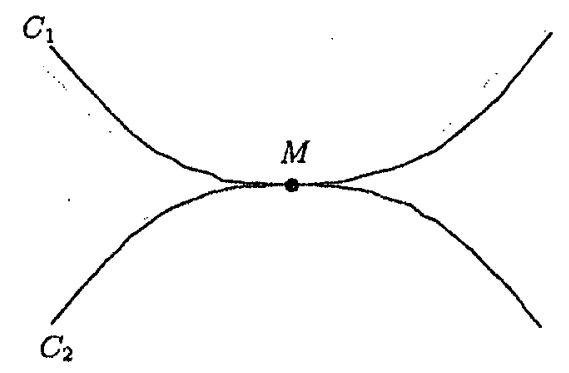

Figure 9: Two curves tangent at regular points

\section{A Some results from algebraic geometry}

Wo: summarise some of the results from algebraic geometry required in this paper. For further information the reader may consult [SK52] or [SR49].

\section{A.1. Plane curves}

A plane curve is given by the zeros of a homogeneous polynomial equation ff(x) in three variables. The polynomial $f(\mathbf{x})$ has a Taylor expansion

$$
f(\mathbf{a}+\mathbf{h})=f(\mathbf{a})+\mathbf{h} \cdot \nabla f(\mathbf{a})+\ldots
$$

at ench point a of $\mathrm{F}^{2}$. If the terms of the Taylor expansion at a vanish up to and including the $r$ th term: then $f(\mathbf{x})$ is sajd to have order $r$ at $\mathbf{a}$. Thus $\mathbf{a}$ is a point of the curve defined by $f(\mathbf{x})$, if and only ir. $r \geq 1$ : If $r \geq 2$ then $f(x)$ is said to have a singularity of order $r$ at $a$ :

Bezout's theorem states that if $f(\mathbf{x}), g(\mathbf{x})$ are plane curves of degrees $m$ and $n$ respectively such that neither is contained in the other, then $f(x)$ and $g(x)$ intersect at exactly $m n$ points. It is important to: count the number of intersections correctly. For example, if $f(x)$ and $g(x)$ are tangent at a point thest this counts 2 towards the total of $m n$ intersections. If $f(\mathbf{x})$. has a singularity of order $h$ at a point, and $g(x)$ has a singularity of order $k$ at the same point, then this counts $h k$ towards the total of intersections. Figure 8 shows the in tersection of two curves at a regular point, figure 9 shows the intersection of two curves tangent. at a regular point, and figure 10 shows the intersection of two curves at a point of order 2 . 


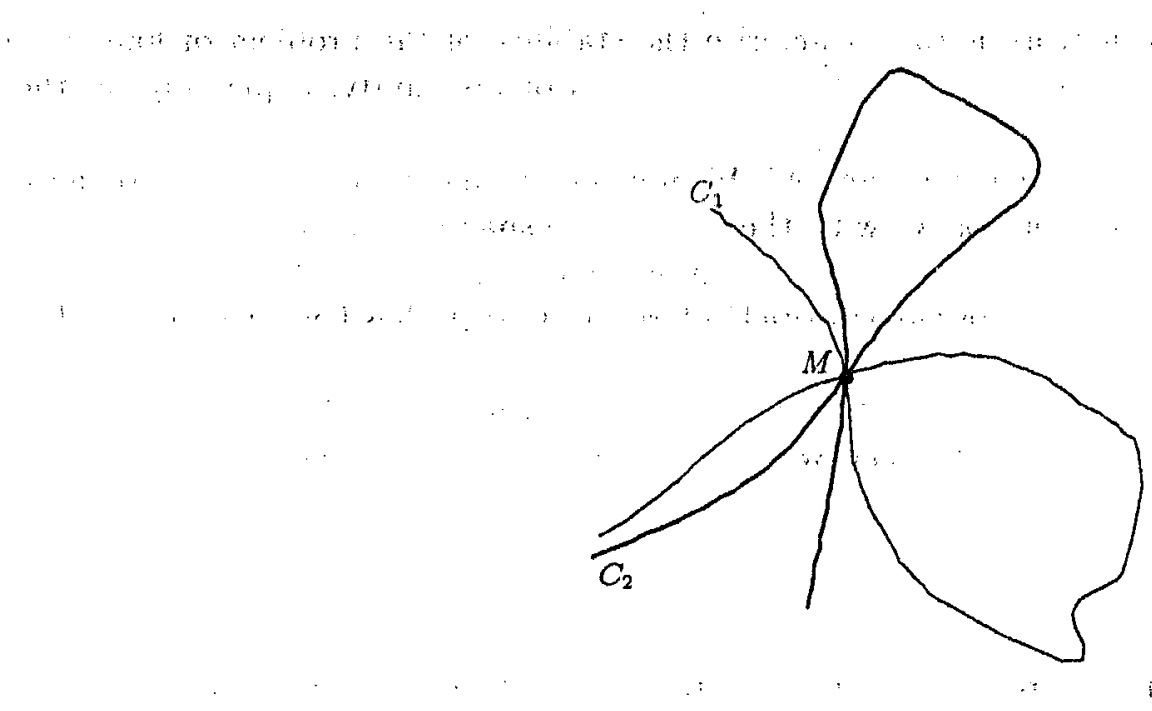

Figure 10: Intersection of two curves at points of order 2

\section{A.2 The degree of an algebraic manifold}

An algebraic manifold $\mathcal{N}$ in projective space $\mathcal{P}^{n}$ is the set of common zeros of a family of polynomials defined on $\mathcal{P}^{m}$. The dimension $n$ of $\mathcal{N}$ is the dimension of the tangent space to $\mathcal{N}$ a.t a general point. Intuitively, the dimension of $\mathcal{N}$ is equal to the minimal number of parameters required to define $\mathcal{N}$. A plane curve is an example of an algebraic manifold of climension 1 . The manifold $\mathcal{M}$ of essential mabrices is an algebraic manifold of dimension 5, since the rotations contribute 3 parameters and the translations, which are only defined up to scale, contribute 2 parameters.

The degree of a plane curve is equal to the degree of the polynomial defining the curve. The degree also has a geometric interpretation as the number of intersections of a general line with the curve. The geometrical definition of degree extends to algebraic manifolds. Let $\mathcal{N}$ be an algebraic manifold in $\mathrm{p}^{m}$. A gencrad linear subspace in $\mathcal{P}^{n}$ of dimension $m-n$ intersects $\mathcal{N}$ at a finite number $d$ of points, and this number $d$ is defined to be the degree of $\mathcal{N}$.

\section{A.3 Resultants}

The resultant of two polynomials arises naturally in connection with the problem of eliminating a common variable. Let $f(x)$ and $g(x)$ be two arbitrary polynomials of degrees $m$ and $n$ respectively, and let

$$
\begin{aligned}
& f(x) \equiv a_{0} x^{n}+a_{1} x^{n-1}+\ldots+a_{n} \\
& g(x) \equiv b_{0} x^{m}+b_{1} x^{m-1}+\ldots+b_{m}
\end{aligned}
$$

The resultant $R(f, g)$ of $f$ and $g$ is the determinant of order $m+n$ :

$$
\left.R(f, g)=\operatorname{det}\left[\begin{array}{ccccccc}
a_{0} & a_{1} & \ldots & a_{n} & 0 & \ldots & 0 \\
0 & a_{0} & \ldots & a_{n-1} & a_{n} & \ldots & 0 \\
\vdots & & & & & & \\
0 & 0 & \ldots & & & & a_{n} \\
b_{0} & b_{1} & \ldots & b_{m} & 0 & \ldots & 0 \\
0 & b_{0} & \ldots & b_{m-1} & b_{m} & \ldots & 0 \\
\vdots & & & & & & \\
0 & 0 & \ldots & & & & b_{m}
\end{array}\right]\right\} m
$$


The fundamental property of $R(f, g)$ is that it is equal to 0 if and only if $a_{0}=b_{0}=0$ or $f(x)$ and $g(x)$ have a non-constant common factor.

Let $f(\mathbf{x}), g(\mathbf{x})$ be homogeneous polynomials of degrees $m$ and $n$ respectively in $\mathbf{x}=\left(x_{1}, x_{2}, x_{3}\right)^{\top}$, and let $x=x_{3}$. Then the coefficients $a_{i}, b_{i}$ of (35) are polynomials in $x_{1}, x_{2}$ and $R(f, g)$ is a homogeneous polynomial in $x_{1}, x_{2}$ of degree $m n$. The roots of $R(f, g)=0$ correspond to the intersections of $f, g$.

\section{B Projective geometry}

We summarise the projective geometry required by Kruppa's method. For further information the reader may consult [SK52]:

\section{B.1 Projective spaces}

A point of $n$ dimensional projective space, $\mathcal{P}^{n}$, is represented by an $n+1$-tuple of coordinates $\mathrm{x}=$ $\left(x_{1}, \ldots x_{n+1}\right)$, where at least one of the $x_{i}$ is non-zero. Two $n+1$-tuples $\left(x_{1}, \ldots x_{n+1}\right)$ and $\left(y_{1}, \ldots y_{n+1}\right)$ represent the same point if and only if there exists a non-zero scalar $\lambda$ such that $x_{i}=\lambda y_{i}$ for $1 \leq i \leq n+1$.

$\mathbf{A}(n+1) \times(n+1)$ matrix $\mathbf{A}$ such that det $(\mathbf{A})$ is different from 0 defines a linear transformation or collineation from $\mathcal{P}^{n}$ into itself. The matrix associated with a given collineation is defined up to a nonzero scale factor, which we usually denote by:

$$
\rho \mathbf{y}=\mathbf{A x}
$$

A projective basis is a set of $n+1$ points of $\mathcal{P}^{n}$ which are linearly independent. For example, the set $\mathbf{e}_{i}=(0, \ldots, 1, \ldots, 0)$, where 1 is in the $i$ th position, is a projective basis, called the standard projective basis. Any point $\mathbf{x}$ of $\mathcal{F}^{n}$ can be described as a linear combination of the standard basis:

$$
\mathbf{x}=\sum_{i=1}^{n+1} x_{i} \mathbf{e}_{i}
$$

The space $\mathcal{P}^{1}$ is known as the projective line. Its standard projective basis is $\mathbf{e}_{1}=(1,0)$, and $\mathbf{e}_{2}=(0,1)$. A point on the line is described by a parameter $\alpha,-\infty \leq \alpha \leq+\infty$ :

$$
\mathrm{x}=c \mathrm{e}_{1}+\mathrm{e}_{2}
$$

The point represented by $e_{1}$ is called the point at infinity of the line $\mathcal{P}^{1}$. It is defined by the linear equation $x_{2}=0$. The reason for this terminology is that if we think of the projective line as containing the usual affine line under the correspondence $\alpha-c \mathbf{e}_{1}+\mathbf{e}_{2}$; then the parameter $\alpha$ gives us a one to one correspondence between the projective and affine lines for all values of $\alpha$ different from $\infty$. The values $\alpha= \pm \infty$ correspond to the point $e_{1}$ outside the affine line but which is the limit of points of the affine line with large values of a.

Let $\mathbf{a}, \mathrm{b}, \mathbf{c}, \mathbf{d}$ be fonr points of $\mathcal{F}^{1}$ with coordinates $\left(\alpha_{a}, 1\right),\left(\alpha_{b}, 1\right),\left(\alpha_{c}, 1\right),\left(\alpha_{d}, 1\right)$, respectively. Then the cross-ratio $\{a, b ; c, d\}$ is clefined to be

$$
\{a, b ; c, d\}=\frac{\left(\alpha_{a}-\alpha_{c}\right)\left(\alpha_{b}-\alpha_{d}\right)}{\left(\alpha_{b}-\alpha_{c}\right)\left(\alpha_{a}-\alpha_{d}\right)}
$$

The significance of the cross-ratio is that it is invariant under invertible linear transformations of $\mathcal{P}^{t}$. In particular, $\{\mathbf{a}, \mathbf{b} ; \mathbf{c}, \mathbf{d}\}$ is independent of the choice of coordinates in $\mathcal{P}^{1}$. 


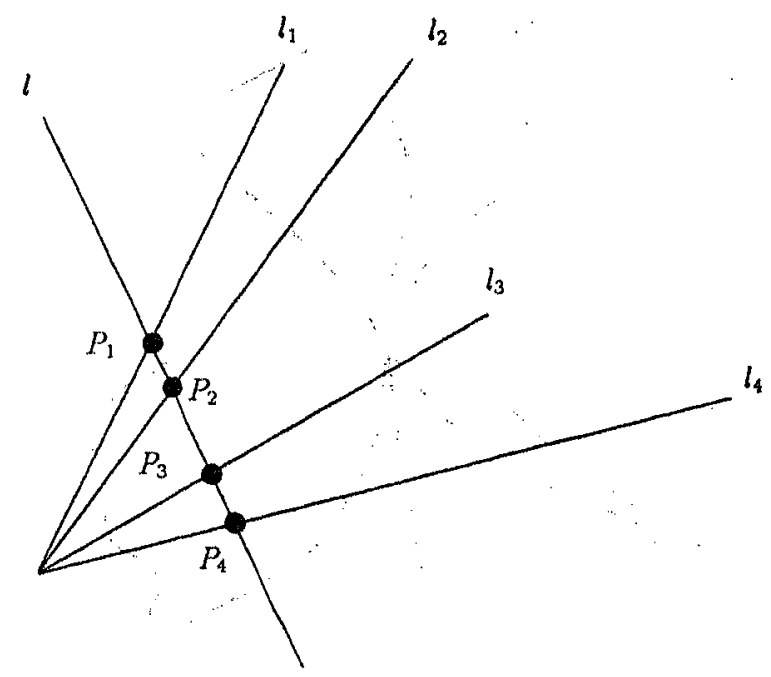

Figure 11: Crossratio of four lines: $\left\{l_{1}, l_{2} ; l_{3}, l_{4}\right\}=\left\{P_{1}, P_{2} ; P_{3}, P_{4}\right\}$

The space $\mathcal{P}^{2}$ is known as the projective plane. A point $\mathbf{x}$ in $\mathcal{P}^{2}$ is defined by three numbers, not all zero, $\left(x_{1}, x_{2}, x_{3}\right)$. In $\mathcal{P}^{2}$, there are other objects than just points, for example lines. A line is also defined by a triplet of numbers $\left(u_{1}, u_{2}, u_{3}\right)$, not all zero. The equation of this line is then:

$$
\sum_{i=1}^{3} u_{i} x_{i}=0
$$

In the standard plojective basis $\left(\mathbf{e}_{1}, \mathbf{e}_{2}, \mathbf{e}_{3}\right)$ of $\mathcal{P}^{2}$. Among all possible lines, the one whose equation is $x_{3}=0$ is called the line at infinity $l_{\infty}$ of $\mathcal{P}^{2}$. Each line of equation (36) intersects $l_{\infty}$ at. the point with coordinates $\left(-u_{2}, u_{1}, 0\right)$ which is its point at infinity.

There are two further points of interest on $l_{\infty}$, called the circular points $i$ and $j$ with coordinates $(1, \pm i, 0)$, where $i=\sqrt{-1}$. They are the intersection of the conic curve $\omega$ of equation $x_{1}^{2}+x_{2}^{2}+x_{3}^{2}$ with $l_{x}$. Their role is very important because they essentially define the metric of the affine plane embedded in $\mathcal{P}^{2}$.

Before we go into this, we need to generalize the notion of cross-ratio introduced" for four points of $\mathcal{F}^{1}$ to four lines of $\mathcal{P}^{2}$ in tersecting at a point. Given four lines $l_{1}, l_{2},, l_{3}, l_{4}$ of $\mathcal{P}^{2}$, intersecting at a point; their cross-ratio $\left\{l_{1}, l_{2} ; l_{3}, l_{4}\right\}$ is defined as the cross-ratio $\left\{\mathbf{p}_{1}, \mathbf{p}_{2} ; \mathbf{p}_{3}, \mathbf{p}_{4}\right\}$ of their four points of intersection with any line $l$ (see figure 11 ), and this is of course independent of the choice of $l$.

Now, the angle $\alpha$ between two lines $l_{1}$ and $l_{2}$ can be defined by considering their point of intersection a and the two lines $i_{a}$ and $j_{a}$ joining a to the circular points $\mathbf{i}$ and $\mathbf{j}$ (see figure 12). The angle is given by Laguerre's formula:

$$
\alpha=\frac{1}{2 i} \log \left(\left\{l_{1}, l_{2} ; i_{a}, j_{a}\right\}\right)
$$

The set of lines in $\mathcal{P}^{2}$ passing through $\mathrm{a}_{;}$fixed point forms a 1 dimensional projective space known an a pencil of lines. Collineations of $\mathcal{P}^{2}$ transform points, lines, and pencils of lines into points, lines, and pencils of lines, and preserve crosstatios.

The space $\mathcal{P}^{3}$ is known as the projective space. A point $\mathbf{x}$ in $\mathcal{P}^{3}$ is clefined by four numbers, $\left(x_{1}, x_{2}, x_{3}, x_{4}\right)$, not all zero. In $\mathcal{P}^{3}$, there are other objects than just points and lines, for example planes. A plane is also 


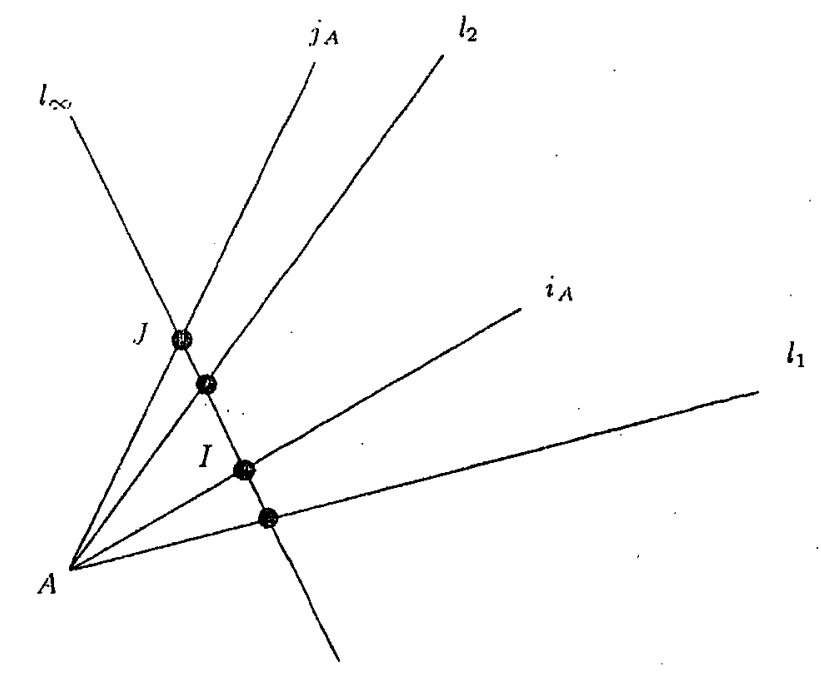

Figure 12 : The angle $\alpha$ between $l_{1}$ and $l_{2}$ is given by Laguerre's formula: $\alpha=\frac{1}{2 i} \log \left(\left\{l_{1}, l_{2}, i_{a}, j_{a}\right\}\right)$.

defined as a four-tuple of numbers $\left(u_{1}, u_{2}, u_{3}, u_{4}\right)$, not all zero. The equation of this plane is then:

$$
\sum_{i=1}^{4} u_{i} x_{i}=0
$$

in the standard projective basis $\left(\mathbf{e}_{1}, \mathbf{e}_{2}, \mathbf{e}_{3}, \mathbf{e}_{4}\right)$ of $\mathcal{P}^{3}$. Among all possible planes, the one whose equation is $x_{4}=0$ is called the plane at. infinity $\pi_{\infty}$ of $\mathcal{P}^{3}$. Each plane of equation (37) intersects the plane at infinity along a line which is its line at infinity.

A special conic $\Omega$ of $\pi_{\infty}$ defined by the two equations:

$$
\sum_{i=1}^{4} x_{i}^{2}=x_{4}=0
$$

plays a special role in $\mathcal{P}^{3}$ and is called the absolute conic. Just as the circular points define the metric of the affine plane embedded in $\mathcal{P}^{2}$, the absolute conic $\Omega$ defines the metric of the affine 3 -space embedded in $P^{3}$.

Before we go into this, we need to generalize the notion of cross-ratio introduced for four points of $\mathbb{P}^{1}$ and four lines of $\mathcal{P}^{2}$, intersecting at a point to four planes of $\mathcal{P}^{3}$ intersecting at a line. Given four planes. $\pi_{1}, \pi_{2}, \pi_{3}, \pi_{4}$ of $\mathcal{P}^{3}$, intersecting at a line $l$, their cross-ratio $\left\{\pi_{1}, \pi_{2} ; \pi_{3}, \pi_{4}\right\}$ is defined as the cross-ratio $\left\{l_{1}, l_{2} ; l_{3}, l_{4}\right\}$ of their four lines of intersection with any plane $\pi$ (see figure 13), and this is of course: independent of the choice of $\pi$.

Now, the angle $\alpha$ between two planes $\pi_{I}$ and $\pi_{2}$ can be defined by considering their line of intersection $l$ and the two planes $i_{l}$ and $j_{l}$ going through $l$ and tangent to the absolute conic $\Omega$ (see figure 14). The arigle is again a simple function of the cross-ratio $\left\{\pi_{1}, \pi_{2} ; i_{l}, j_{l}\right\}$ :

$$
\alpha=\frac{1}{2 i} \log \left(\left\{\pi_{1}, \pi_{2} ; i_{l}, j_{l}\right\}\right)
$$

Similarly, in $P^{3}$. the set of planes containing a fixed line forms a pencil of planes. Collineations of: $P^{3}$ transform points, lines, planes, and pencils of planes into points, lines, planes and pencils of planes; and preserve crossratios. 


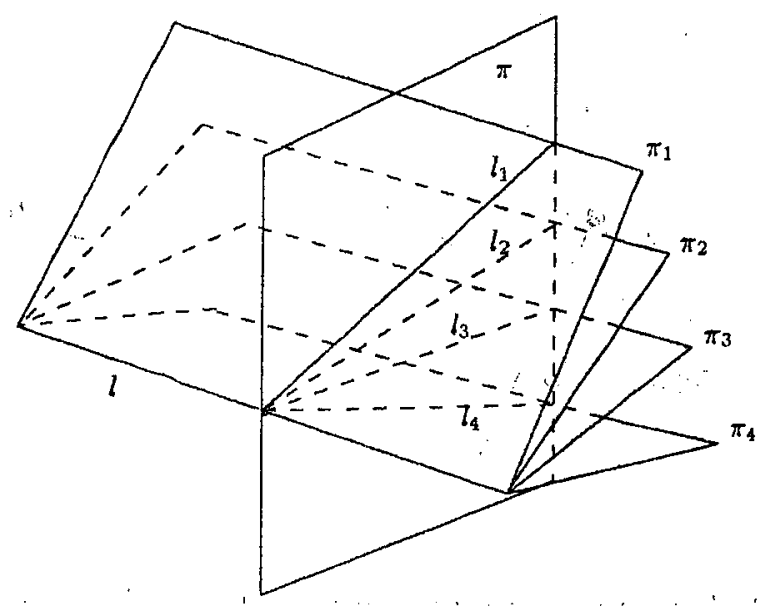

Figure 13: Crossratio of four planes: $\left\{\pi_{1}, \pi_{2} ; \pi_{3}, \pi_{4}\right\}=\left\{l_{1}, l_{2} ; l_{3}, l_{4}\right\}$

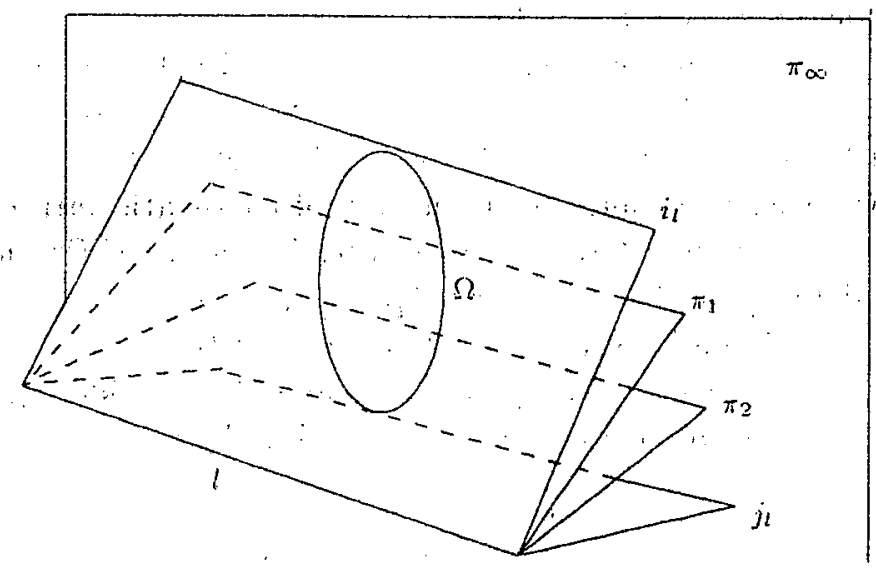

Figure 14: The angle between the two plaies $\pi_{1}$ and $\pi_{2}$ is a simple function of the crossitio $\left(\pi_{1}, \pi_{2} ; i_{1}, j_{l}\right)$, where $i_{l}$ and $j_{l}$ are the two tangent, planes from $l$ to the absolute conic $\Omega$ 
* 4 A quadratic trantormation is an inventible transformation between projective planes defined by polynomials of degree two If $\mathbf{x}-\mathbb{X}$ is a quadratic transformation then coordinates in the two projective planes can always be chosen such that .

$$
\Sigma \mathbf{x}=\left(x_{2} x_{3}, x_{3} x_{1}, x_{1} x_{2}\right)^{\top}
$$

A quadratic transformationis undefined at exactly three non-collineari points, known as fundamental points. The fundamental points of $\Sigma$ are $(1,0,0),(0,1,0),(0,0,1)$. A quadratic transformation has an inverse which is also a quadratic transformation. $\Sigma$ is self-inverse. 


\begin{tabular}{|c|c|c|c|c|}
\hline $\begin{array}{l}\text { Solution } \\
\text { number }\end{array}$ & axis of rotation & rotation angle & axis of translation & feasibility \\
\hline 1 & {$\left[\begin{array}{lll}-.416 & -.801 & -.430\end{array}\right]^{1}$} & $171.11 \mathrm{deg}$ & {$\left[\begin{array}{lll}.420 & .338 & -.842\end{array}\right]^{2}$} & no \\
\hline 2 & {$\left[\begin{array}{lll}-.788 & .558 & -.261\end{array}\right]^{T^{\prime}}$} & $170.46 \mathrm{deg}$ & same & yes \\
\hline 3 & {$\left[\begin{array}{lll}.696 & .714 & .073\end{array}\right]^{T^{\prime}}$} & $179.23 \mathrm{deg}$ & {$\left[\begin{array}{lll}.325 & -.531 & -.783\end{array}\right]^{t}$} & no \\
\hline 4 & {$\left[\begin{array}{llll}.534 & -.586 & .610\end{array}\right]^{\prime \prime}$} & $155.74 \mathrm{deg}$ & same & no \\
\hline 5 & {$\left[\begin{array}{lll}-.814 & -.578 & .061\end{array}\right]^{T}$} & $179.89 \mathrm{deg}$ & {$\left[\begin{array}{lll}-.671 & -.726 & -.149\end{array}\right]^{\mathrm{J}}$} & no \\
\hline 6 & {$\left[\begin{array}{lll}.451 & -.555 & .699\end{array}\right]^{1}$} & $33.75 \mathrm{deg}$ & same & no \\
\hline 7 & {$\left[\begin{array}{lll}-.542 & .588 & -.600\end{array}\right]^{4}$} & $172.62 \mathrm{deg}$ & {$\left[\begin{array}{lll}-.487 & .275 & .829\end{array}\right]^{\prime \prime}$} & no \\
\hline 8 & {$\left[\begin{array}{lll}-.684 & -.724 & -.084\end{array}\right]^{T}$} & $171.77 \mathrm{deg}$ & same & no \\
\hline 9 & {$\left[\begin{array}{lll}-.561 & -.292 & -.774\end{array}\right]^{1 *}$} & $177.01 \mathrm{deg}$ & {$\left[\begin{array}{lll}.545 & .276 & .792\end{array}\right]^{2}$} & no \\
\hline 10 & {$\left[\begin{array}{lll}.818 & -.392 & .422\end{array}\right]^{3}$} & $4.45 \mathrm{deg}$ & same & no \\
\hline 11 & {$\left[\begin{array}{llll}.693 & -.028 & -.720\end{array}\right]^{\mathrm{T}^{\prime}}$} & 174.93 deg & {$\left[\begin{array}{lll}.626 & .219 & .748\end{array}\right]^{2}$} & no \\
\hline 1.2 & {$\left[\begin{array}{lll}-.111 & .984 & -.137\end{array}\right]^{11}$} & $167.29 \mathrm{deg}$ & same & yes \\
\hline 13 & {$\left[\begin{array}{lll}.110 & -.983 & .145\end{array}\right]^{\prime \prime}$} & $175.48 \mathrm{deg}$ & {$\left[\begin{array}{lll}-.742 & -.138 & -.656\end{array}\right]^{t^{\prime}}$} & no \\
\hline 14 & {$\left[\begin{array}{lll}-.694 & .030 & .719\end{array}\right]^{\prime \prime}$} & $175.30 \mathrm{deg}$ & same & no \\
\hline 15 & {$\left[\begin{array}{lll}.077 & .170 & -.082\end{array}\right]^{12}$} & $176.57 \mathrm{deg}$ & {$\left[\begin{array}{lll}-.068 & -.160 & .985\end{array}\right]^{\prime \prime}$} & no \\
\hline 16 & {$\left[\begin{array}{lll}-.374 & .148 & .915\end{array}\right]^{2}$} & $3.79 \mathrm{deg}$ & same & no \\
\hline 17 & {$\left[\begin{array}{lll}.415 & .798 & .438\end{array}\right]^{10}$} & $177.33 \mathrm{deg}$ & {$\left[\begin{array}{lll}-.419 & -.141 & .897\end{array}\right]^{\prime \prime}$} & no \\
\hline 18 & {$\left[\begin{array}{lll}.791 & -.555 & .250\end{array}\right]^{I}$} & $167: 87 \mathrm{deg}$ & same & yes \\
\hline 19 & {$\left[\begin{array}{lll}.248 & .279 & -.928\end{array}\right]^{7}$} & $176.85 \mathrm{deg}$ & {$\left[\begin{array}{lll}-.216 & -.276 & .936\end{array}\right]^{\prime}$} & $\begin{array}{l}\text { no } \\
\end{array}$ \\
\hline 20 & {$\left[\begin{array}{lll}-.261 & .552 & .792\end{array}\right]^{2}$} & $4.89 \mathrm{deg}$ & same & nio \\
\hline
\end{tabular}

Table 2: 10 real solutions, 3 feasible 


\begin{tabular}{|c|c|}
\hline Points in retina 1 & Points in retina 2 \\
\hline$\therefore=\left[\begin{array}{lll}1000 & 2000 & 1000\end{array}\right]^{2}$ & $\mathbf{a}^{\prime}=\left[\begin{array}{lll}2232 & 134 & 1001\end{array}\right]^{I^{\prime}}$ \\
\hline $\mathbf{b}=\left[\begin{array}{lll}1414 & -1414 & 1414\end{array}\right]^{J^{\prime}}$ & $\mathbf{b}^{\prime}=\left[\begin{array}{lll}-518 & -1931 & 1415\end{array}\right]^{\prime}$ \\
\hline $\mathbf{c}=\left[\begin{array}{lll}-1732 & 0 & 1732\end{array}\right]^{T}$ & $c^{\prime}=\left[\begin{array}{lll}-860 & 1500 & 1733\end{array}\right]^{\prime \prime}$ \\
\hline $\mathrm{d}=\left[\begin{array}{lll}2000 & 1000 & 3000\end{array}\right]^{\prime \prime}$ & $d^{\prime}=\left[\begin{array}{ll}1866 & -1232\end{array}\right.$ \\
\hline $\mathrm{e}=\left[\begin{array}{lll}-1000 & -1000 & 2000\end{array}\right]^{T}$ & $e^{\prime}=\left[\begin{array}{lll}-1366 & 366 & 2001\end{array}\right]^{\prime \prime}$ \\
\hline
\end{tabular}

Table 3: Coordinates of matched points yielding 8 real solutions 


\begin{tabular}{|c|c|c|}
\hline $\begin{array}{l}\text { Solution } \\
\text { number }\end{array}$ & $\begin{array}{c}\text { axis of rotation } \\
\text { axis of translation }\end{array}$ & rotation angle \\
\hline $\begin{array}{c}\text { real } \\
\text { solution }\end{array}$ & $\begin{array}{c}{\left[\begin{array}{lll}0 & 0 & .9999999995\end{array}\right]^{T}} \\
{\left[\begin{array}{lll}.009999000150 & .009999000150 & -.9999000150\end{array}\right]^{I}}\end{array}$ & $60.00145558 \mathrm{deg}$ \\
\hline 1 & $\begin{array}{c}{\left[\begin{array}{lll}-.002487442670 & -.001059610836 & .99999963465\end{array}\right]^{T}} \\
{\left[\begin{array}{lll}.8307803010 & -.2844253432 & -.4784415488\end{array}\right]^{T}}\end{array}$ & $59.92096991 \mathrm{deg}$ \\
\hline 2 & $\begin{array}{c}{\left[\begin{array}{ccc}.0003116759826 & -.0003594703083 & .9999998852\end{array}\right]^{T}} \\
{\left[\begin{array}{lll}.3624612636 & .6367204572 & -.6805945131\end{array}\right]^{t}}\end{array}$ & $60.00521800 \mathrm{deg}$ \\
\hline 3 & 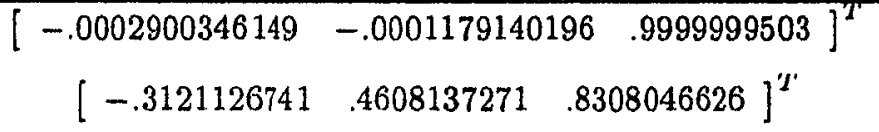 & $60.00822116 \mathrm{deg}$ \\
\hline 4 & 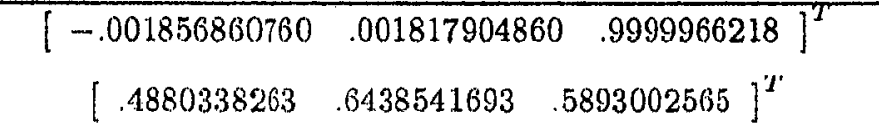 & $60.08204432 \mathrm{deg}$ \\
\hline 5 & $\begin{array}{c}{\left[\begin{array}{ccc}.5500156220 * 10^{-5} & .0001613512601 & .9909999872\end{array}\right]^{T}} \\
{\left[\begin{array}{lll}.1993944775 & .1011375165 & .9746861263\end{array}\right]^{T^{T}}}\end{array}$ & $60.00124982 \mathrm{deg}$ \\
\hline 6 & $\begin{array}{c}{\left[\begin{array}{lll}.0003638641 .453 & .0002492030755 & .9999999031\end{array}\right]^{T}} \\
{\left[\begin{array}{lll}.6760324163 & .05249643690 & .7349995214\end{array}\right]^{x}}\end{array}$ & $59.98729079 \mathrm{deg}$ \\
\hline 7 & 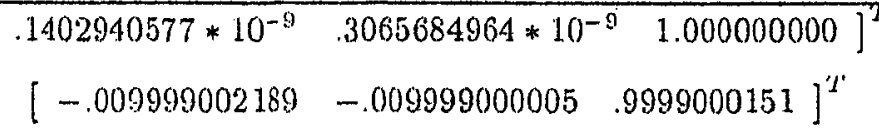 & $60.00145558 \mathrm{deg}$ \\
\hline 8 & $\begin{array}{c}{\left[\begin{array}{lll}.00008888119202 & -.0001711784862 & .0999099803\end{array}\right]^{T}} \\
{\left[\begin{array}{lll}-.1341471213 & -.3176202417 & .9386809529\end{array}\right]^{I^{\prime}}}\end{array}$ & $60.00003843 \mathrm{deg}$ \\
\hline
\end{tabular}

Table $4: 8$ real solutions, 8 feasible 


\section{B.2 The role of the absolute conic in Kruppa's method}

One of the key points in Kruppa's method is the introduction of the rigidity constraint in a natural way using the device of the absolute conic $\Omega$ defined in the previous section. The effectiveness of Kruppa's method depends on the fact that the images $\omega, \omega^{\prime}$ of $\Omega$ determine and are determined by the camera calibration. To prove this, we show first that $\omega$ (and $\omega^{\prime}$ ) is independent of the camera position. Let a camera (c, $\Pi$ ) undergo a rigid motion $W$, where $\mathbf{c}$ is the optical center and $I I$ the retinal plane. The new position of the camera is $(W(\mathbf{c}), W(\Pi))$. Let $\omega_{1}$ and $\omega_{2}$ be the images of $\Omega$ in $\Pi$ and $W(\Pi)$ respectively. Then $\omega_{2}$, regarded as a conic in $W(\Pi)$, is the image of $W(\Omega)=\Omega$, thus $\omega_{1}=\omega_{2}$ as required. It follows that $\omega$ is determined by the camera calibration since we can put the camera in any convenient position and calculate $\omega$ by using the known camera calibration and the known position of $\Omega$.

We prove the converse. ie that $\omega$ determines the camera calibration. Let $r, s$ be two projection rays meeting the retinal plane at $\mathbf{m}_{1}, \mathbf{n}_{1}$ and meeting the plane at infinity at $\mathbf{m}_{2} \mathbf{n}_{2}$, respectively. Let $\iota$ and $\varsigma$ be the two intersections of $\left\langle\mathrm{m}_{2}, \mathbf{n}_{2}\right\rangle$ with $\Omega$. Then the angle between $r$ and $s$ is given by the Laguerre formula $\frac{1}{2 i} \log \left(\left\{\mathbf{m}_{2}, \mathbf{n}_{2} ; \iota, \varsigma\right\}\right)$. The cross-ratio $\left\{\mathbf{m}_{2}, \mathbf{n}_{2} ; \iota, \varsigma\right\}$ is preserved under the projection $M$ to the retinal plane, thus the angle between $r$ and $s$ is given by:

$$
\left.\frac{1}{2 i} \log \left(\left\{\mathbf{m}_{1}, \mathbf{n}_{1} ; M(\iota), M(\varsigma)\right\}\right)\right)
$$

Now $M(\iota)$ and $M(\varsigma)$ are the intersection points of $\left\langle\mathrm{m}_{1}, \mathrm{n}_{1}>\right.$ with $\omega$ thus $\omega$ determines the angle between $r$ and $s$, thus the camera calibration. 
